\title{
mTORC2 promotes type I insulin-like growth factor receptor and insulin receptor activation through the tyrosine kinase activity of mTOR
}

Yancun Yin ${ }^{1}$, Hui Hua ${ }^{2}$, Minjing $\mathrm{Li}^{3}$, Shu Liu ${ }^{1}$, Qingbin Kong ${ }^{1}$, Ting Shao ${ }^{1}$, Jiao Wang ${ }^{4}$, Yuanming Luo ${ }^{5}$, Qian Wang ${ }^{5}$, Ting Luo ${ }^{6}$, Yangfu Jiang ${ }^{1}$

${ }^{I}$ State Key Laboratory of Biotherapy, Section of Oncogene, West China Hospital, Sichuan University, Chengdu, Sichuan 610041, China; ${ }^{2}$ Laboratory of Stem Cell Biology, West China Hospital, Sichuan University, Chengdu, Sichuan 610041, China; ${ }^{3}$ Medicine and Pharmacy Research Center, Binzhou Medical University, Yantai, Shandong 264003, China; ${ }^{4}$ School of Basic Medicine, Chengdu University of Traditional Chinese Medicine, Chengdu, Sichuan 610075, China; ${ }^{5}$ State Key Laboratory of Microbial Resources, Institute of Microbiology, Chinese Academy of Sciences, Beijing 100101, China; ${ }^{6}$ Cancer Center, West China Hospital, Sichuan University, Chengdu, Sichuan 610041, China

Mammalian target of rapamycin (mTOR) is a core component of raptor-mTOR (mTORC1) and rictor-mTOR (mTORC2) complexes that control diverse cellular processes. Both mTORC1 and mTORC2 regulate several elements downstream of type I insulin-like growth factor receptor (IGF-IR) and insulin receptor (InsR). However, it is unknown whether and how mTOR regulates IGF-IR and InsR themselves. Here we show that mTOR possesses unexpected tyrosine kinase activity and activates IGF-IR/InsR. Rapamycin induces the tyrosine phosphorylation and activation of IGF-IR/InsR, which is largely dependent on rictor and mTOR. Moreover, mTORC2 promotes ligand-induced activation of IGF-IR/InsR. IGF- and insulin-induced IGF-IR/InsR phosphorylation is significantly compromised in rictor-null cells. Insulin receptor substrate (IRS) directly interacts with SIN1 thereby recruiting mTORC2 to IGF-IR/InsR and promoting rapamycin- or ligand-induced phosphorylation of IGF-IR/InsR. mTOR exhibits tyrosine kinase activity towards the general tyrosine kinase substrate poly(Glu-Tyr) and IGF-IR/InsR. Both recombinant mTOR and immunoprecipitated mTORC2 phosphorylate IGF-IR and InsR on Tyr1131/1136 and Tyr1146/1151, respectively. These effects are independent of the intrinsic kinase activity of IGF-IR/InsR, as determined by assays on kinase-dead IGF-IR/InsR mutants. While both rictor and mTOR immunoprecitates from rictor ${ }^{+/+} \mathrm{MCF}-10 \mathrm{~A}$ cells exhibit tyrosine kinase activity towards IGF-IR and InsR, mTOR immunoprecipitates from rictor ${ }^{-/-}$MCF-10A cells do not induce IGF-IR and InsR phosphorylation. Phosphorylation-deficient mutation of residue Tyr1131 in IGF-IR or Tyr1146 in InsR abrogates the activation of IGF-IR/InsR by mTOR. Finally, overexpression of rictor promotes IGF-induced cell proliferation. Our work identifies mTOR as a dual-specificity kinase and clarifies how mTORC2 promotes IGF-IR/InsR activation.

Keywords: mTOR; mTORC2; insulin receptor; insulin-like growth factor receptor; protein kinase Cell Research (2016) 26:46-65. doi:10.1038/cr.2015.133; published online 20 November 2015

\section{Introduction}

The mechanistic target of rapamycin (mTOR) is a

Correspondence: Yangfu Jiang

Tel: 86-28-85164044 ; Fax: 86-28-85164046

E-mail: jyangfu@scu.edu.cn

Received 6 May 2015; revised 15 September 2015; accepted 8 October 2015; published online 20 November 2015 serine/threonine kinase that integrates signals from nutrients, stress and growth factors, thereby regulating cell metabolism, proliferation, autophagy and migration $[1,2]$. mTOR interacts with several proteins to form two complexes termed mTOR complex 1 (mTORC1) and mTOR complex 2 (mTORC2). Raptor and PRAS40 are specific to $\mathrm{mTORC} 1$, while rictor, $\mathrm{mSIN} 1$, and protor $1 / 2$ are specific components in mTORC2. mTORC1 mainly acts as an energy and redox sensor that controls protein synthe- 
sis through two well-characterized targets, p70-S6 kinase 1 (S6K1) and eukaryotic initiation factor 4E-binding protein 1 [2]. In addition, mTORC1 indirectly phosphorylates carbamoyl-phosphate synthetase 2 through S6K1 thereby stimulating the synthesis of nucleotides [3, 4]. On the other hand, mTORC2 regulates the cytoskeleton through its stimulation of F-actin stress fibers and Rho GTPases [5]. mTORC2 also phosphorylates the serine/ threonine protein kinase $\mathrm{Akt} / \mathrm{PKB}$ on the serine residue S473 [6]. Although rapamycin can induce Akt activation through derepression of $\mathrm{mTORC} 2$, prolonged rapamycin treatment may inhibit mTORC2 assembly and Akt activation in certain cell types [7]. In addition, mTORC2 was shown to regulate protein kinase $\mathrm{C} \alpha$ and serum/ glucocorticoid-induced protein kinase $1[8,9]$. Recently, it has been found that mTORC1 and its downstream effector S6K1 negatively regulate mTORC2 via the phosphorylation of rictor and SIN1 [10-12], suggesting a regulatory link between these two complexes. In addition, prolonged treatment with rapamycin, an antibiotic product that predominantly inhibits $\mathrm{mTORC} 1$, leads to ERK1/2 phosphorylation [13]. The mechanisms underlying the induction of Akt and ERK1/2 phosphorylation by rapamycin may involve the stabilization of insulin receptor substrate 1 (IRS1), a critical signal transducer downstream of insulin receptor (InsR) and type I insulin-like growth factor receptor (IGF-IR) but upstream of both Akt and ERK1/2 [14].

It is well known that mTORC1 senses signal from growth factors, such as insulin and insulin-like growth factors (IGF-I and IGF-II). IGF-IR is a transmembrane tyrosine kinase that is phosphorylated and activated upon binding to IGF, thereby acting as a growth promoter. Type II IGF receptor (IGF-IIR), however, lacks intrinsic kinase activity and negatively regulates circulating and local levels of IGF-II by targeting it to lysosomes for degradation, thereby acting as a growth inhibitor [15]. InsR or IGF-IR exists in dimer. In addition, a half InsR and a half IGF-IR can form heterodimeric receptor that binds to IGF-I but poorly binds to insulin [16]. Signaling through IGF-IR/InsR is largely mediated by adaptor molecules, including IRS1, IRS2, Shc, and Grb10. IRS1 and IRS2 contain multiple tyrosine phosphorylation motifs that serve as docking sites for SH2 domain-containing proteins which in turn activate signaling pathways such as PI3K and MAPK/ERK cascades [17]. While mTORC1 acts as an effector downstream of IGF/insulin signaling and elicits feedback inhibition of IRS1 and stabilization of Grb10 [18, 19], mTORC2 may positively regulate IGF signaling by phosphorylating IGF-II mRNA-binding protein to promote IGF-II translation [20, 21]. In addition, mTORC2 disruption in mice leads to insulin resistance
$[22,23]$. mTORC2 also negatively feeds back to IRS1 via Fbw8-mediated degradation [24]. Despite a handful of reports that provide support for the involvement of $\mathrm{mTOR}$ in IGF/insulin signaling, IGF-IR and InsR have not been considered as membrane receptors that are directly regulated by $\mathrm{mTOR}$.

Here, we identify mTOR as a dual-specificity kinase that phosphorylates and activates IGF-IR/InsR. Except for the well-known serine/threonine kinase activity, mTOR exhibits tyrosine kinase activity. In cell-free system, mTOR directly phosphorylates tyrosine residues in IGF-IR/InsR. In cells, the phosphorylation and activation of IGF-IR/InsR by mTOR is mediated by rictor. Furthermore, IRS1 and IRS2 recruit mTORC2 to IGF-IR/InsR and promote IGF-IR/InsR phosphorylation. Phosphorylation of Y1131 in IGF-IR and Y1146 in InsR is essential for the activation of IGF-IR/InsR by mTOR.

\section{Results}

Inhibition of mTORC1 leads to IGF-IR/InsR phosphorylation and activation

IGF-IR and InsR share a triple tyrosine cluster (Y1131/1135/1136 for IGF-IR, and Y1146/1150/1151 for InsR) within the kinase domain, which is known as the earliest major sites of autophosphorylation necessary for kinase activation $[25,26]$. To determine the effect of mTORC1 inhibition on IGF-IR/InsR phosphorylation, we treated hepatoma cells HepG2 with rapamycin, followed by western blot analysis of IGF-IR/InsR phosphorylation at the triple tyrosine cluster. Treatment of HepG2 cells with rapamycin resulted in a decrease in $\mathrm{S} 6 \mathrm{~K} 1$ phosphorylation (Figure 1A). Also, phosphorylation of IRS1 at the S6K1-dependent sites (S636/639) was inhibited (Figure 1A). However, treatment with rapamycin led to an increase in phosphorylation of IGF-IR/InsR at the triple tyrosine cluster (Figure 1A). In the meantime, treatment with rapamycin promoted Akt and ERK1/2 phosphorylation (Figure 1A). Similar results were obtained in another hepatoma cell line, SMMC-7721 (Supplementary information, Figure S1A). The phosphorylation of IGF-IR/ InsR, Akt and ERK1/2 promoted by rapamycin increased over time (Figure 1B and Supplementary information, Figure S1B). In addition, rapamycin promoted IGF-IR/ InsR phosphorylation in HeLa and Chang liver cells (Supplementary information, Figure S1C).

Since the antibodies may react with both phosphorylated IGF-IR and InsR, we investigated whether rapamycin induced both IGF-IR phosphorylation and InsR phosphorylation. First, we overexpressed GFP-tagged InsR in HepG2 cells. Rapamycin promoted the phosphorylation of ectopic InsR and endogenous IGF-IR/ 

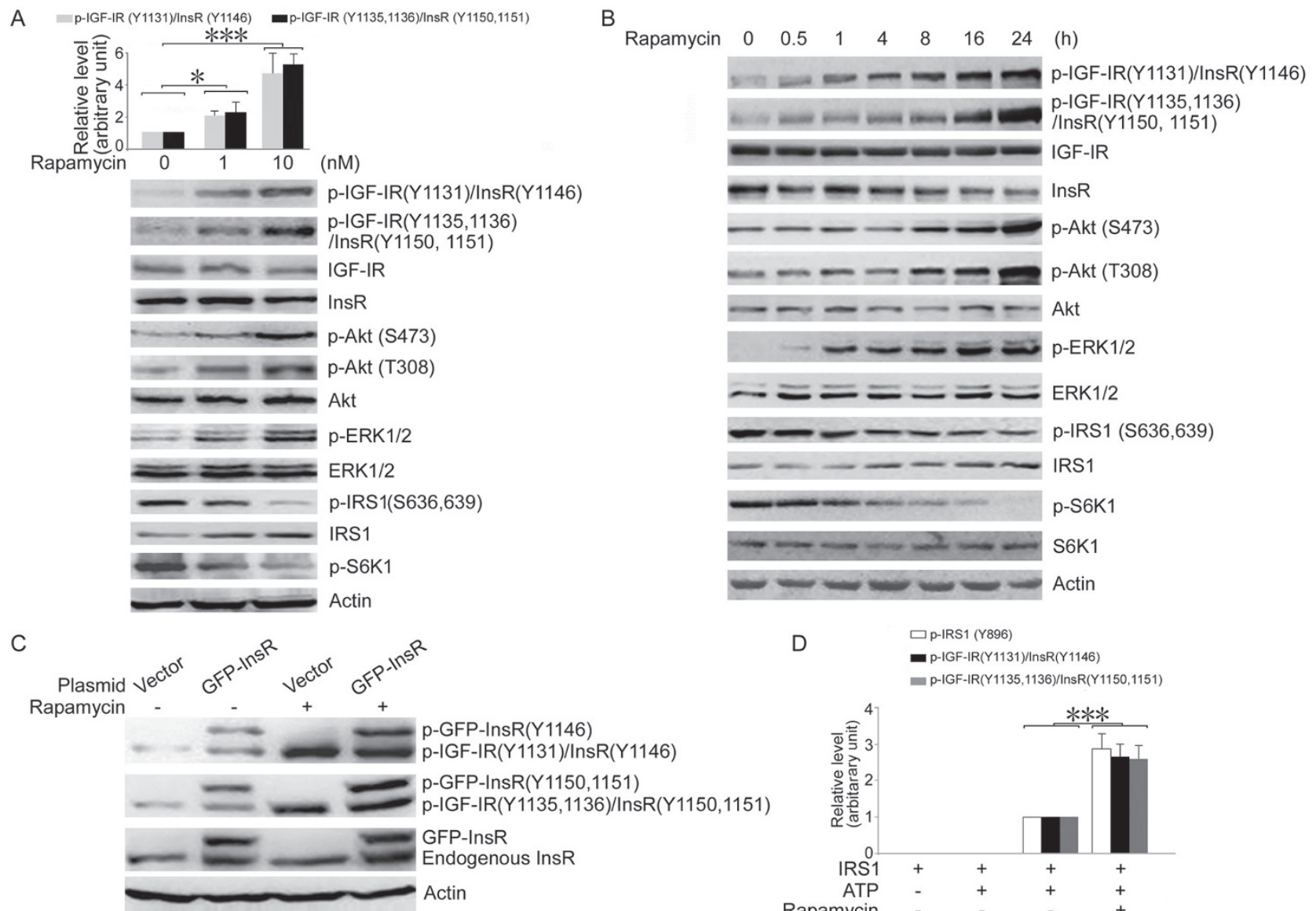

E
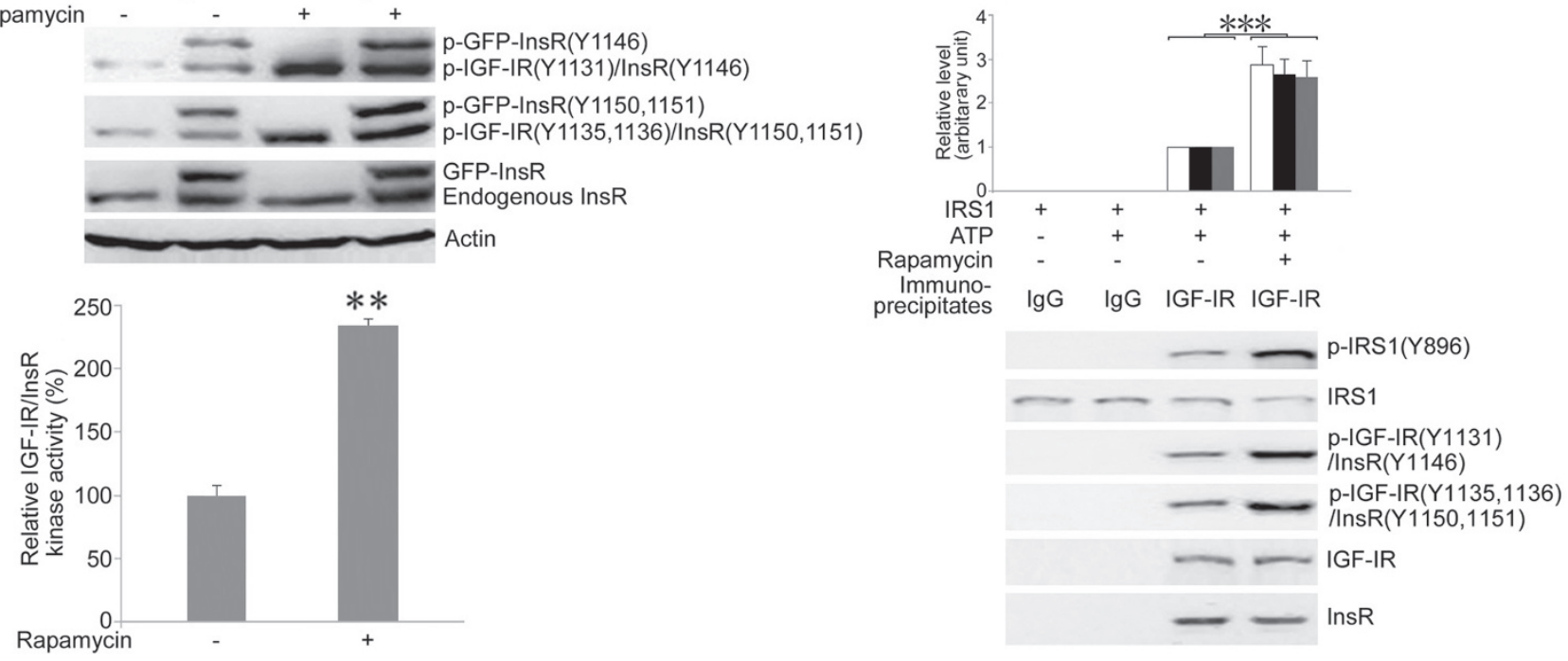

Figure 1 Rapamycin potentiates IGF-IR/InsR phosphorylation and activation. (A) HepG2 cells were treated with or without rapamycin for $24 \mathrm{~h}$, followed by western blot analysis of indicated proteins. Values represent mean $\pm \mathrm{SD}$. $* P<0.05$; ***P< 0.001. (B) HepG2 cells were treated with rapamycin for the indicated period, followed by western blot analysis. (C) Endogenous and GFP-tagged InsR and their phosphorylation in HepG2 cells treated with or without 10 nM rapamycin for 24 h. (D) The immunoprecipitated IGF-IR/InsR from rapamycin-treated or untreated HepG2 cells was incubated with 50 ng recombinant IRS1 in a kinase assay system, followed by western blot analysis and quantification. Values represent mean \pm SD. ${ }^{* * *} P$ $<0.001$. (E) The tyrosine kinase activity of immunoprecipitated IGF-IR/InsR from HepG2 cells treated with or without $10 \mathrm{nM}$ rapamycin for $24 \mathrm{~h}$ was examined by in vitro kinase assay, using a synthetic peptide (300 $\mathrm{ng})$ as the substrate. The relative kinase activity is plotted. The IGF-IR/InsR kinase activity in untreated cells is set as $100 \%$. Values represent mean \pm SD $(n=4)$. $* * P<0.01$. A representative of two or more experiments is shown.

InsR (Figure 1C). In addition, the GFP-tagged InsR was transfected into MDA-MB-453 cells without endogenous IGF-IR/InsR expression, followed by treatment with rapamycin. Rapamycin promoted InsR phosphorylation in MDA-MB-453 cells (Supplementary information, Figure S1D). Second, a mammalian expression plasmid for IGF-IR was transfected into MDA-MB-453 cells, followed by treatment with rapamycin. Treatment with 
rapamycin resulted in an increase in IGF-IR phosphorylation (Supplementary information, Figure S1E). These data demonstrate that rapamycin can promote both IGFIR and InsR phosphorylation. While rapamycin could induce Akt phosphorylation in the absence of IGF-IR/ InsR, it failed to induce ERK $1 / 2$ phosphorylation in the absence of IGF-IR/InsR (Supplementary information, Figure S1D, S1E). Overexpression of IGF-IR or InsR further enhanced the induction of Akt phosphorylation and potentiated the induction of ERK1/2 phosphorylation by rapamycin (Supplementary information, Figure S1D, S1E). Thus, the promotion of ERK1/2 phosphorylation by rapamycin is due to the activation of IGF-IR/ InsR, while the activation of IGF-IR/InsR contributes, in part, to the promotion of Akt phosphorylation by rapamycin. Moreover, rapamycin poorly promoted IGFIR/InsR phosphorylation upon serum starvation, while it significantly promoted IGF-IR/InsR phosphorylation after resupply of serum, IGF-1 or insulin (Supplementary information, Figure S1F).

To determine whether rapamycin stimulates the tyrosine kinase activity of IGF-IR/InsR, we immunoprecipitated IGF-IR/InsR from cells treated with or without rapamycin, followed by in vitro kinase assay using either recombinant IRS 1 or synthetic peptide as substrate. Since IGF-IR can heterodimerize with InsR, immunoprecipitation of IGF-IR co-precipitated InsR. Compared to the immunoprecipitated IGF-IR/InsR from rapamycin-untreated cells, the immunoprecipitated IGF-IR/InsR from rapamycin-treated cells exhibited higher levels of tyrosine phosphorylation and induced higher levels of IRS1 phosphorylation (Figure 1D). The kinase activity of IGF-IR/InsR towards the peptide substrate for IGF-IR/ InsR was significantly higher in rapamycin-treated cells than in rapamycin-untreated cells (Figure 1E). These data demonstrate that treatment with rapamycin leads to an increase in the tyrosine kinase activity of IGF-IR/InsR.

To investigate whether raptor or S6K1 knockdown has effects similar to rapamycin, we examined the phosphorylation of IGF-IR/InsR, Akt and ERK1/2 in raptor or S6K1 knockdown cells. Reduced expression of target proteins was confirmed. Knockdown of raptor or S6K1 led to IGF-IR/InsR phosphorylation in HepG2 cells (Supplementary information, Figure S1G). Despite reducing S6K1 phosphorylation to the similar extent as raptor siRNA, mTOR knockdown failed to increase IGF-IR phosphorylation (Supplementary information, Figure S1G). Rather, mTOR or rictor knockdown abrogated IGF-IR/ InsR phosphorylation resulting from raptor or S6K1 depletion (Supplementary information, Figure $\mathrm{S} 1 \mathrm{H}$ ). The contrasting effects of raptor and mTOR knockdown on IGF-IR/InsR phosphorylation suggest that mTOR con- tributes to IGF-IR/InsR phosphorylation induced by raptor or S6K1 depletion.

mTORC2 promotes rapamycin- and ligand-induced IGF$I R / I n s R$ phosphorylation

The above data demonstrate that mTOR is involved in the phosphorylation and activation of IGF-IR/InsR resulting from inhibition of mTORC1 pathway. Previous studies have revealed that $\mathrm{S} 6 \mathrm{~K} 1$ phosphorylates rictor and SIN1 thereby negatively regulating mTORC2 activity $[11,12]$. Therefore, treatment with rapamycin or downregulation of raptor and S6K1 may paradoxically activate mTORC2. To determine whether mTORC2 is involved in rapamycin-induced IGF-IR/InsR phosphorylation, the effect of rictor or mTOR knockdown on rapamycin-induced IGF-IR/InsR phosphorylation was examined. Either mTOR or rictor knockdown inhibited the induction of IGF-IR/InsR phosphorylation by rapamycin (Figure 2A, 2B). Moreover, rapamycin failed to promote IGF-IR/InsR phosphorylation in rictor-null MCF-10A cells (Figure 2C).

To determine whether rictor and mTOR are involved in the induction of IGF-IR phosphorylation by its ligand, HepG2 and SMMC-7721 cells were transfected by rictor or mTOR siRNA, followed by treatment with IGF-I and detection of IGF-IR phosphorylation. Knockdown of mTOR or rictor reduced ligand-induced IGF-IR, Akt and ERK1/2 phosphorylation (Figure 2D, 2E). In addition, knockdown of rictor or mTOR led to a decrease in insulin-induced InsR phosphorylation (Figure 2F, 2G). Knockdown of mTOR or rictor with another siRNA also inhibited the induction of IGF-IR/InsR phosphorylation by rapamycin or IGF-I (Supplementary information, Figure S2A, S2B). Moreover, overexpression of rictor enhanced rapamycin- or IGF-I-induced IGF-IR/InsR phosphorylation (Figure $2 \mathrm{H}$ and Supplementary information, Figure S2C). Restoration of rictor by siRictor-resistant rictor construct rescued the defect in IGF-IR/InsR phosphorylation due to rictor depletion (Figure $2 \mathrm{H}$ and Supplementary information, Figure S2C). Notably, the rictor-mTOR complex remained stable after treatment of HepG2 cells with rapamycin for $36 \mathrm{~h}$ (Supplementary information, Figure S2D). Also, the mTORC2 activity increased over time, as determined by phosphorylation of its substrate, Akt2 (Supplementary information, Figure S2D). Finally, IGF-I and insulin induced IGF-IR/InsR phosphorylation in rictor-null MCF-10A cells to a much lesser extent than in wild-type cells (Figure 2I). The induction of InsR and ERK1/2 phosphorylation by insulin was significantly enhanced by expression of rictor in rictor-null MCF-10A cells (Supplementary information, Figure S2E). 


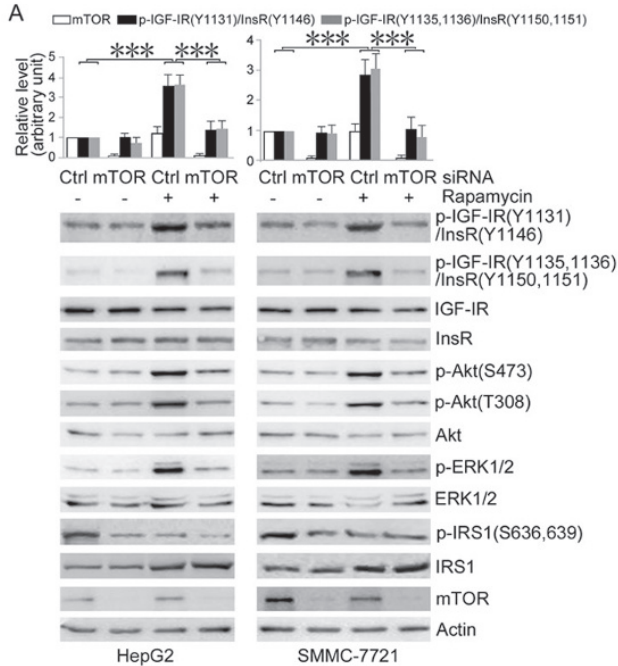

D

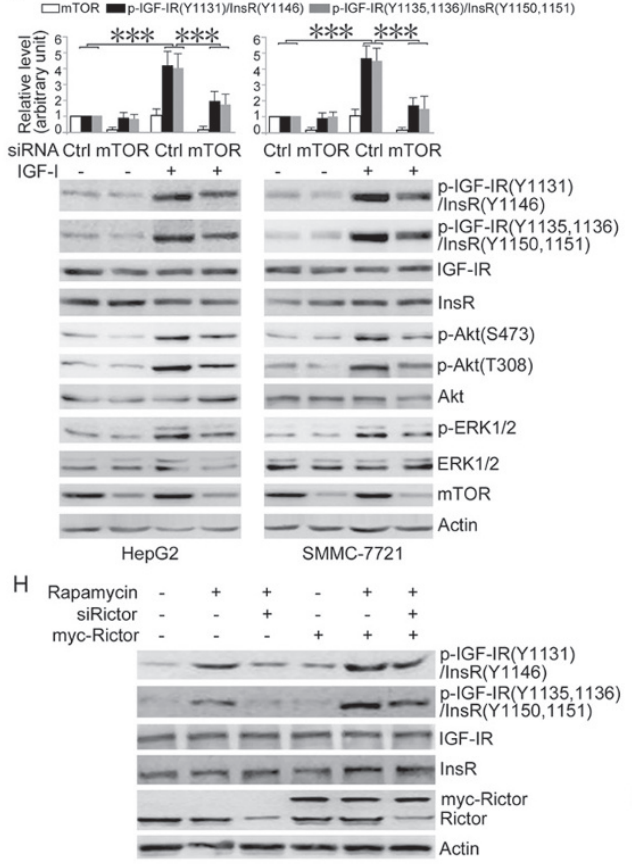

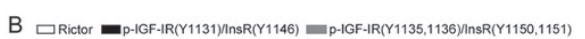

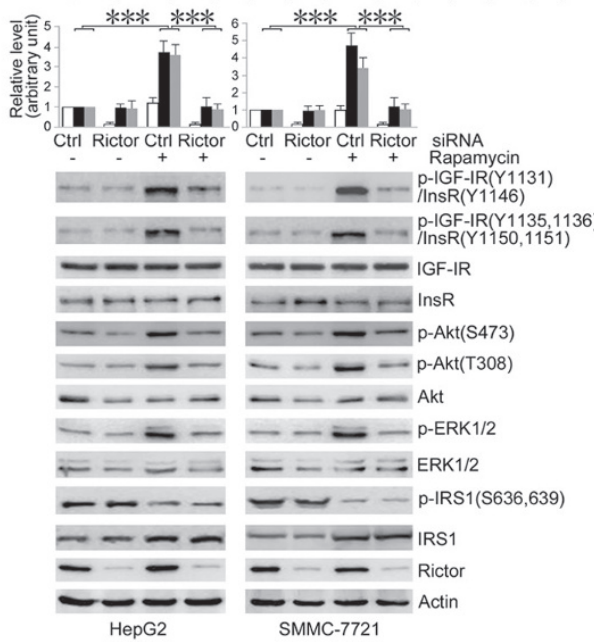

E

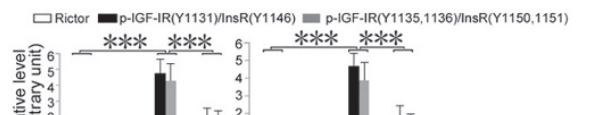

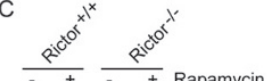

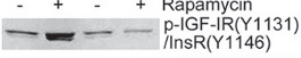

- - D-IGF-IR(Y1135,1136)

- $-\cdots-I G F-I R$

- - - InsR

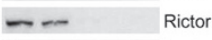

I- ${ }_{\text {Actin }}$

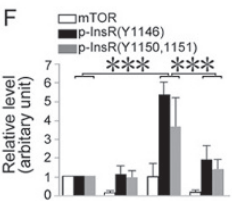

CtrI mTOR Ctrl mTOR siRNA $\therefore \perp^{+}{ }^{+}-\ln R(Y 1146)$ - - - ${ }^{\mathrm{p}-\ln \mathrm{n} R}$ $-\infty \mathrm{InsR}$ - - - $\mathrm{mTOR}^{-}$ $-\simeq$ - Actin

G
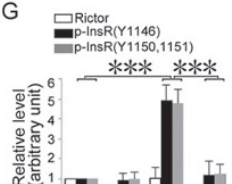

Ctrl Rictor Ctrl Rictor siRNA

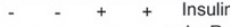
$-1=-\begin{gathered}\text { P-InsR } \\ (\text { Y1146) }\end{gathered}$
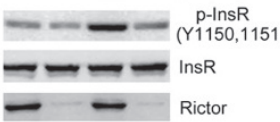

$-$

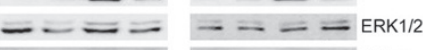

- - - - - - - Rictor

$-\underset{\text { HepG2 }}{-}-\underset{\text { SMMC-7721 }}{- \text { Actin }}$

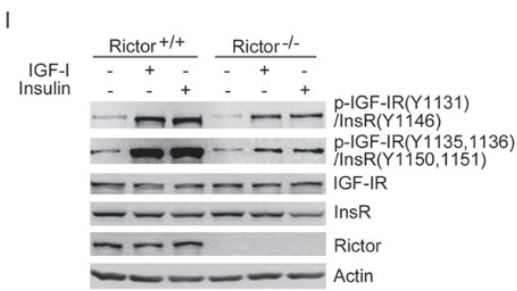

Figure 2 Rictor and mTOR promote rapamycin- and ligand-induced phosphorylation and activation of IGF-IR/InsR. (A) HepG2 and SMMC-7721 cells were transfected with negative control siRNA or MTOR siRNA, and treated with or without 10 $\mathrm{nM}$ rapamycin for $24 \mathrm{~h}$, followed by western blot analysis of the indicated proteins. Values represent mean $\pm \mathrm{SD}$. $* * * P<0.001$. (B) HepG2 and SMMC-7721 cells were transfected with negative control siRNA or rictor siRNA, and treated with or without $10 \mathrm{nM}$ rapamycin for $24 \mathrm{~h}$, followed by western blot analysis. Values represent mean $\pm \mathrm{SD}$. $* * * P<0.001$. (C) MCF-10A (rictor ${ }^{+/}$) and rictor-null MCF-10A $\left(\right.$ (ictor $^{-1-}$ ) cells were treated with or without $10 \mathrm{nM}$ rapamycin for $24 \mathrm{~h}$, followed by western blot analysis of the indicated proteins. (D) HepG2 and SMMC-7721 cells were transfected with negative control siRNA or mTOR siR$\mathrm{NA}$, and treated with or without $1 \mathrm{nM}$ IGF-I for $30 \mathrm{~min}$, followed by western blot analysis. Values represent mean \pm SD. $* * * P$ $<0.001$. (E) HepG2 and SMMC-7721 cells were transfected with negative control siRNA or rictor siRNA, and treated with or without $1 \mathrm{nM}$ IGF-I for $30 \mathrm{~min}$, followed by western blot analysis. Values represent mean $\pm \mathrm{SD}$. ***P<0.001. (F) HepG2 cells were transfected with negative control siRNA or mTOR siRNA, and treated with or without $10 \mu \mathrm{g} / \mathrm{mL}$ insulin for $30 \mathrm{~min}$, followed by western blot analysis. Values represent mean $\pm \mathrm{SD}$. ${ }^{* * *} P<0.001$. (G) HepG2 cells were transfected with negative control siRNA or rictor siRNA, and treated with or without $10 \mu \mathrm{g} / \mathrm{mL}$ insulin for $30 \mathrm{~min}$, followed by western blot analysis. Values represent mean $\pm \mathrm{SD}$. ${ }^{* * *} P<0.001$. (H) The effects of overexpression of siRictor-resistant rictor construct (myc-Rictor) on rapamycin-induced IGF-IR/InsR phoshorylation in HepG2 cells transfected with or without siRictor. Values represent mean \pm SD. $* * P<0.01 ; * * * P<0.001$. (I) MCF-10A ( rictor $^{+/+}$) and rictor-null MCF-10A $\left(\right.$rictor $^{-/-}$) cells were treated with or without 1 $\mathrm{nM}$ IGF-I and $10 \mu \mathrm{g} / \mathrm{mL}$ insulin for $30 \mathrm{~min}$, followed by western blot analysis of the indicated proteins. A representative of two or more experiments is shown. 
$I R S 1 / 2$ recruit $m T O R C 2$ to $I G F-I R / I n s R$ and promote $I G F-I R / I n s R$ phosphorylation

The above results suggest that $\mathrm{mTORC} 2$ is a positive regulator of IGF-IR/InsR. We then determined whether endogenous rictor and mTOR form a complex with IGFIR/InsR. Immunoprecipitation of IGF-IR or InsR from HepG2 cells co-precipitated both rictor and mTOR, while raptor was not detected in the immunoprecipitates (Figure $3 \mathrm{~A})$. Conversely, immunoprecipitation of either rictor or mTOR co-precipitated IGF-IR and InsR (Figure 3A). These data suggest that IGF-IR and InsR form complexes with mTORC2 but not mTORC1. IRS1 and IRS2, two known IGF-IR/InsR substrates, were detected in IGFIR, InsR, rictor and mTOR immunoprecipitates (Figure 3A). Conversely, immunoprecipitation of IRS1 or IRS2 co-precipitated IGF-IR, InsR, rictor and mTOR (Figure $3 \mathrm{~A})$. Mass spectrometric analysis of rictor immunoprecipitates validated that IGF-IR, InsR, IRS1 and IRS2 are interacting components of rictor (Supplementary information, Table S1). Meanwhile, immunoprecitation of rictor co-preciptated mTOR, IGF-IR, InsR, IRS1 and IRS2 in MCF-10A cells but not in rictor-null MCF-10A cells, confirming the specificity of rictor immunoprecipitation (Figure 3B). Knockdown of rictor reduced the interaction between IGF-IR and mTOR (Figure 3C), suggesting that rictor is required for the integrity of mTOR-IGF-IR complex. Also, knockdown of rictor led to a decrease in the interaction between mTOR and InsR (Figure 3C). Importantly, knockdown of IRS $1 / 2$ reduced the interaction between rictor-mTOR and IGF-IR/InsR (Figure 3D, 3E), suggesting that the adaptor proteins IRS1 and IRS2 are necessary for recruiting rictor-mTOR to IGF-IR/InsR. However, pull-down assay did not demonstrate direct interaction between IRS1 and rictor (data not shown). Instead, pull-down assay with purified IRS1 and SIN1 demonstrated a direct interaction between these proteins, suggesting that SIN1 mediates the interaction between IRS and mTORC2 (Figure 3F). Moreover, SIN1 knockdown impaired the interaction among IGF-IR/InsR, rictor and mTOR (Figure 3G). The knockdown efficiency and the expression of multiple proteins were confirmed (Supplementary information, Figure S3). Since treatment with rapamycin resulted in an increase in the levels of IRS1/2 (Supplementary information, Figure S4A), it also elevated the abundance of IGF-IR/InsR-IRS1/2-rictormTOR complexes (Supplementary information, Figure S4B, S4C). Consistent with the inhibition of mTORC1 by AMP-activated protein kinase (AMPK), an important energy sensor, treatment of HepG2 cells with the AMPK agonist AICAR increased the formation of IGF-IR-rictormTOR complexes (Supplementary information, Figure S4D). In addition, treatment of HepG2 cells with insulin led to an increase in InsR-IRS1/2-rictor-mTOR complexes (Supplementary information, Figure S4E). Finally, immunoprecipitation of IGF-IR from mouse liver lysates co-precipitated rictor, mTOR and InsR, indicating that IGF-IR/InsR-rictor-mTOR complexes exist in vivo (Supplementary information, Figure S4F).

Consistent with the notion that IRS $1 / 2$ recruit mTORC2 to IGF-IR/InsR, knockdown of both IRS1 and IRS2 blunted the promotion of IGF-IR/InsR, Akt and ERK1/2 phosphorylation by rapamycin and ligand (Figure 3H-3J). Similar effects were detected when both IRS1 and IRS2 were depleted by another set of siRNA (Supplementary information, Figure S5). These data suggest that IRS1/2 not only serve as adaptor proteins for downstream effectors, but also recruit regulators of IGF-IR/ InsR.

The kinase activity of mTOR is required for its promotion of IGF- and insulin-induced IGF-IR/InsR phosphorylation and activation

To determine whether mTOR kinase activity is required for its promotion of IGF-induced phosphorylation of the triple tyrosine cluster of IGF-IR and InsR, we treated HepG2 and SMMC-7721 cells with Torin2, a potent ATP-competitive mTOR inhibitor, followed by treatment with IGF-I or insulin. Torin2 inhibited IGF-Iinduced IGF-IR/InsR, Akt and ERK1/2 phosphorylation (Figure 4A, Supplementary information, Figure S6A). Also, the induction of InsR phosphorylation by insulin was inhibited by Torin2 (Supplementary information, Figure S6B, S6C). These data indicate that the kinase activity of mTOR is necessary for promotion of IGF-IR and InsR phosphorylation.

We next examined the kinase activity of endogenous raptor-mTOR complex and rictor-mTOR complex towards recombinant IGF-IR and InsR by in vitro kinase assays. mTOR, rictor and raptor immunoprecipitates were collected from MDA-MB-453 breast cancer cells that are negative for IGF-IR and InsR so that there was not IGF-IR/InsR in these immunopreciptates. Rictor and mTOR immunoprecipitates phosphorylated the triple tyrosine cluster of IGF-IR/InsR, whereas the raptor immunoprecipitates did not induce IGF-IR/InsR phosphorylation (Figure 4B, 4C and Supplementary information, Figure S7). Torin2 inhibited the phosphorylation of IGF-IR/InsR by rictor and mTOR immunoprecipitates (Figure 4B, 4C). Moreover, immunoprecipitates of rictor and mTOR phosphorylated the triple tyrosine cluster in kinase-dead (KD) IGF-IR (K1003R)/InsR (K1018A; Figure 4D, 4E). In contrast, the mTOR-related kinase ATM did not induce IGF-IR/InsR phosphorylation, while autophosphorylation of ATM was detected (Figure 4D, 
A
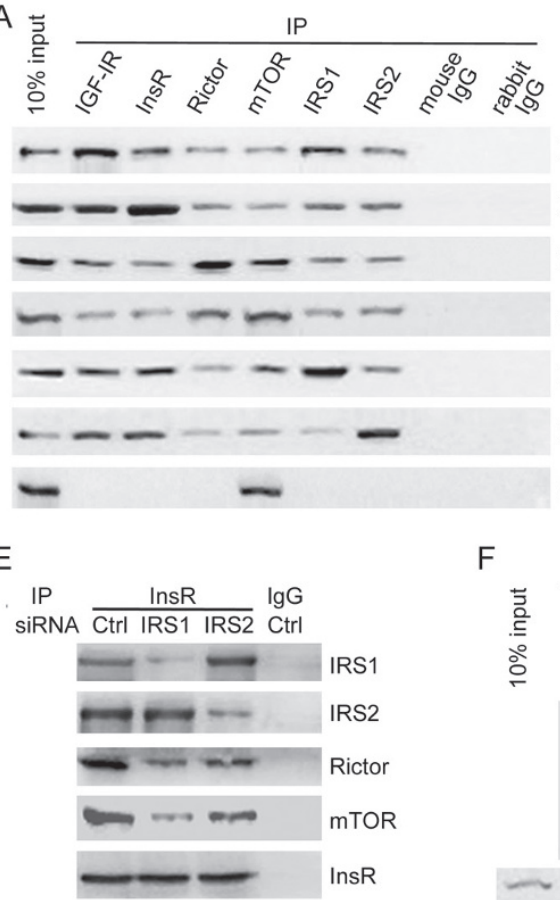

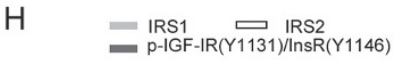

- p-IGF-IR $(Y 1135,1136) / \operatorname{Ins} R(Y 1150,1151)$

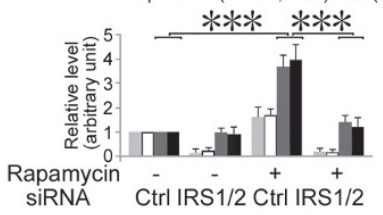

siRNA Ctrl IRS $1 / 2$ Ctrl IRS $1 / 2$

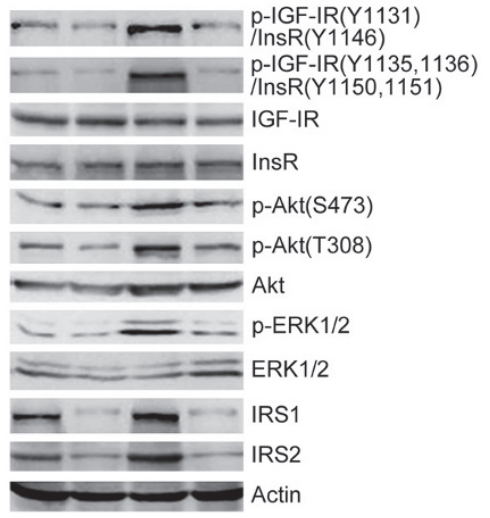

B

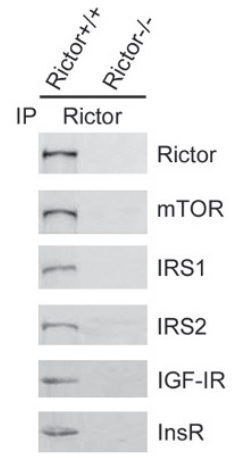

C
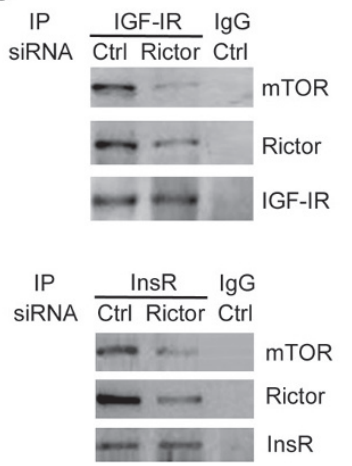

D

$\underset{\text { IP }}{\text { IPA }} \frac{\text { IGF-IR }}{\text { Ctrl IRS1 IRS2 }}$ CtrI - - IRS1
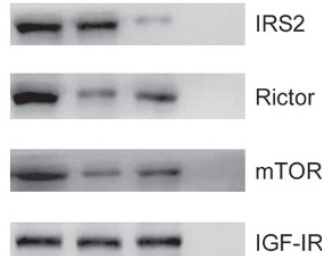

Raptor

F

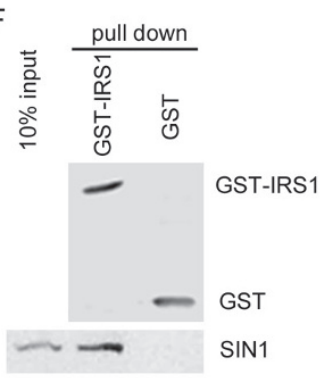

G

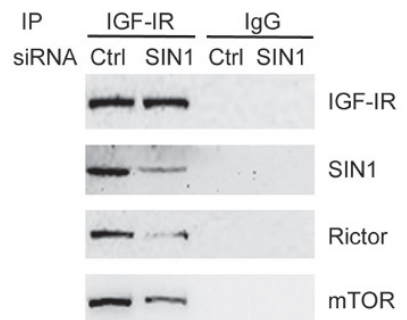

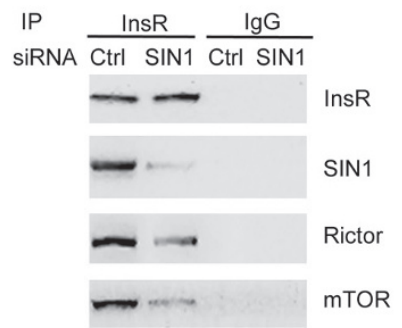

$\operatorname{IP}_{\text {SiRNA }} \frac{\operatorname{lnsR}}{\text { Ctrl SIN1 }} \frac{\lg \mathrm{G}}{\text { Ctrl SIN1 }}$

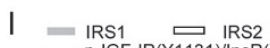

- $p-I G F-I R(Y 1131) / \ln R(Y 1146)$

- ${ }^{p-I G F-I R(Y 1135,1136) / I n s R(Y 1150,1151)}$

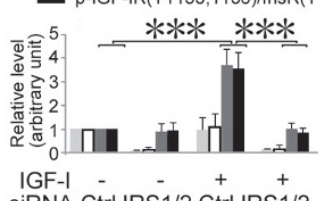

siRNA Ctrl IRS $1 / 2 \mathrm{Ctrl}$ IRS $1 / 2$

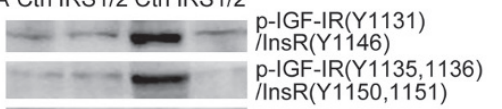

$=--1$ IGF-IR

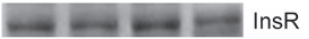

-

$\longrightarrow$ p-Akt(T308)

$\longrightarrow$ Akt

$=\ldots$ p-ERK $1 / 2$

$=$ ERK $1 / 2$

- $=$ IRS1

- . - IRS2

Actin
$\mathrm{J}=\mathrm{IRS} 1 \square \mathrm{IRS} 2$

= ${ }^{\mathrm{p}-\operatorname{InsR}(\mathrm{I} F-\operatorname{In} 1146)}$

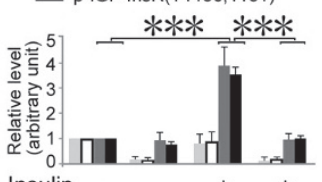

$\begin{array}{llll}\text { Insulin } & - & - & + \\ \text { siRNA Ctrl IRS } 1 / 2 & + & + \\ \text { Ctrl IRS } 1 / 2\end{array}$

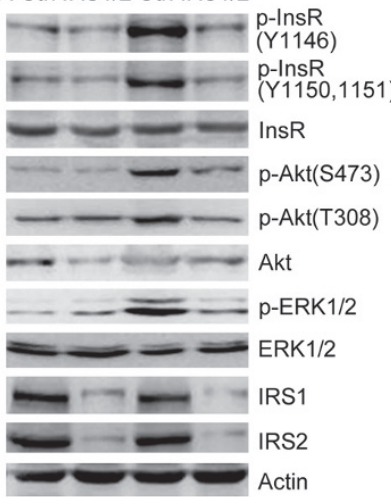

Figure 3 IRS1/2 recruit rictor-mTOR to IGF-IR/InsR and promote their phosphorylation. (A) Immunoprecipitation of IGF-IR, InsR, rictor, mTOR, IRS1 and IRS2, and western blot analysis of the indicated proteins. (B) Immunoprecipitation of rictor from MCF-10A $\left(\right.$ rictor $^{++}$) and rictor null MCF-10A cells $\left(\right.$rictor $^{-/-}$). (C) Immunoprecipitation of IGF-IR and InsR from HepG2 cells transfected with negative control siRNA or rictor siRNA. (D) Immunoprecipitation of IGF-IR from HepG2 cells transfected with negative control siRNA, IRS1 or IRS2 siRNA, and western blot analysis. (E) Immunoprecipitation of InsR from HepG2 cells transfected with negative control siRNA, IRS1 or IRS2 siRNA, and western blot analysis. (F) GST pull-down assay of purified GST-IRS1 and SIN1. (G) Immunoprecipitation of IGF-IR and InsR from HepG2 cells transfected with negative control siRNA or SIN1 siRNA. (H) The effects of simultaneous knockdown of both IRS1 and IRS2 on rapamycin-induced IGF-IR/InsR phosphorylation. (I) The effects of simultaneous knockdown of both IRS1 and IRS2 on IGF-I-induced IGF-IR/InsR phosphorylation. (J) The effects of simultaneous knockdown of both IRS1 and IRS2 on insulin-induced InsR phosphorylation. Values represent mean $\pm \mathrm{SD}$. $* * * P<0.001$. A representative of three experiments is shown. 

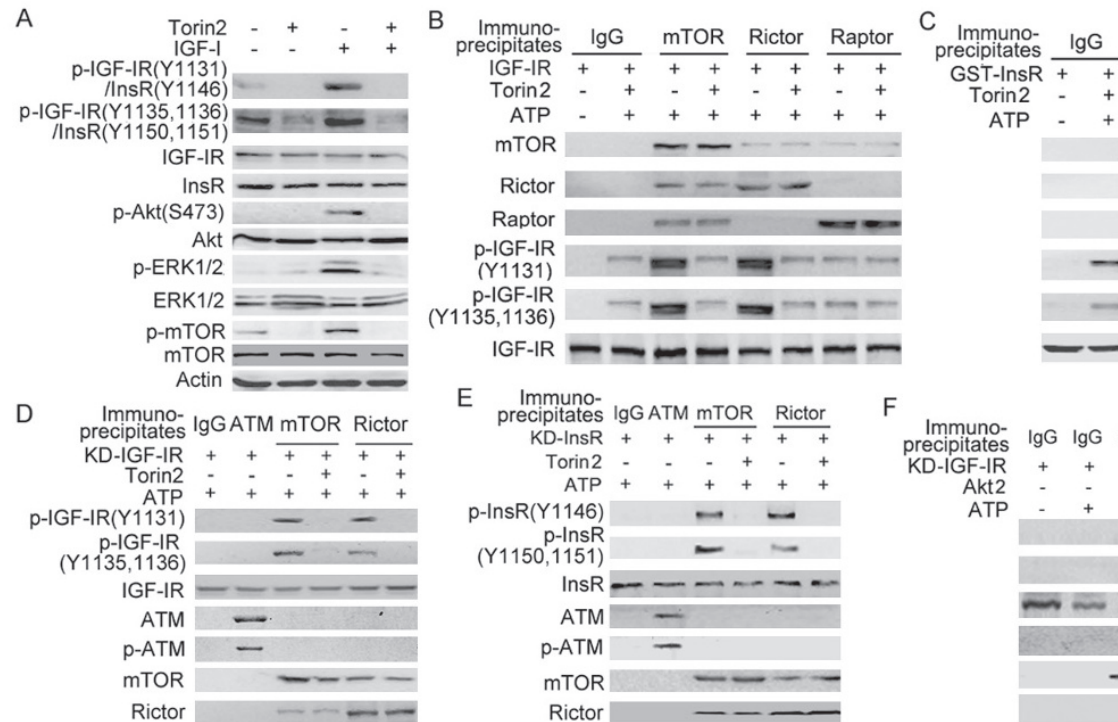

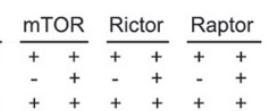

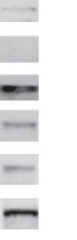

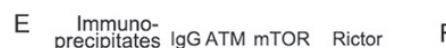
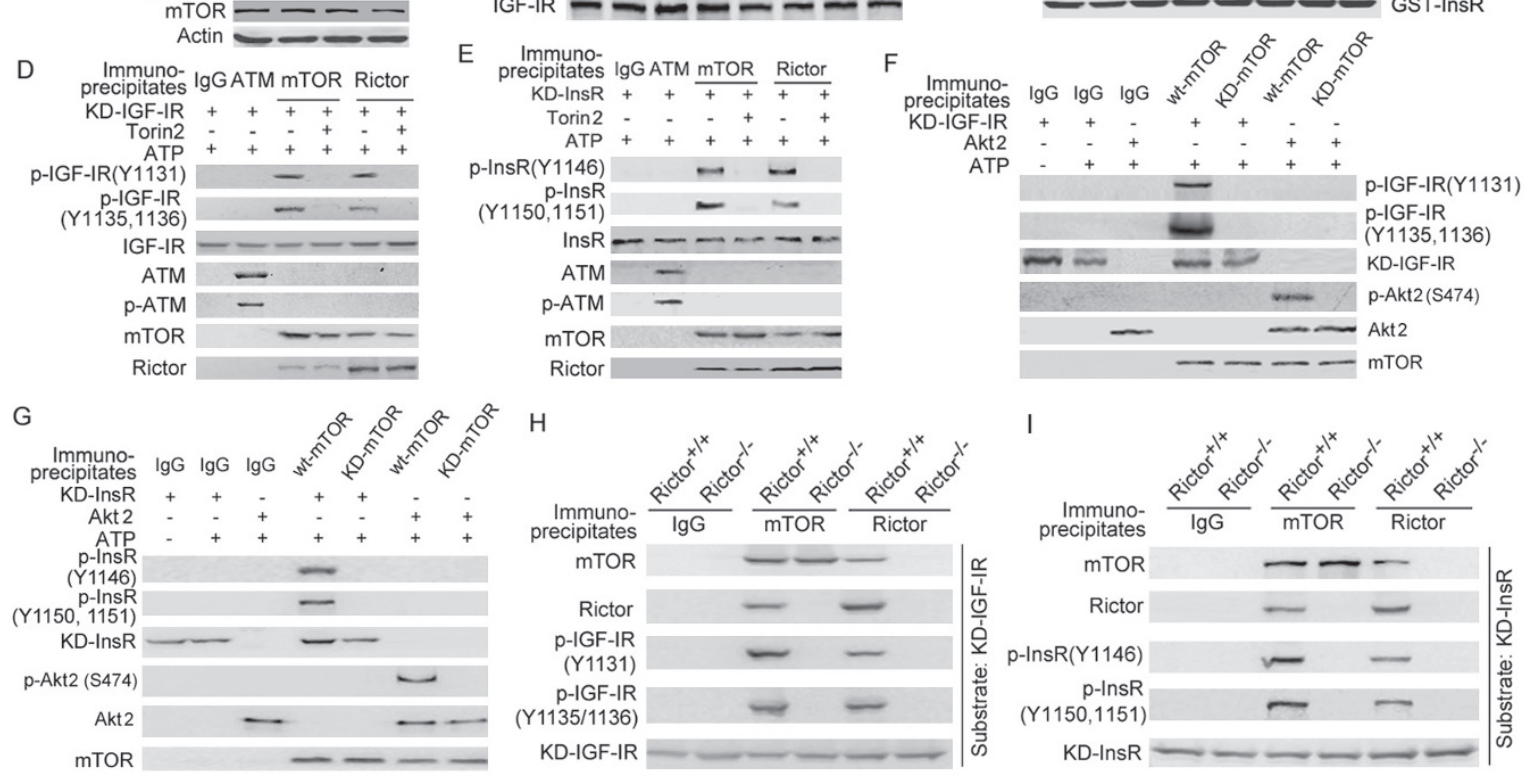

Figure 4 mTOR promotes IGF-IR/InsR phosphorylation and activation. (A) Western blot analysis of IGF-IR/InsR, Akt, ERK1/2 and mTOR phosphorylation in HepG2 cells that were pre-treated with or without $2 \mathrm{nM}$ Torin2 for $4 \mathrm{~h}$, and treated with or without $1 \mathrm{nM}$ IGF-I for $30 \mathrm{~min}$, or $10 \mu \mathrm{g} / \mathrm{mL}$ insulin for $30 \mathrm{~min}$. Values represent mean \pm SD. ${ }^{* * *} P<0.001$. (B) MDA-MB-453 cell lysates (IGF-IR negative and InsR negative) were subjected to immunoprecipitation of raptor, rictor and mTOR complexes. Immunoprecipitated raptor, rictor and mTOR complexes were incubated with recombinant IGF-IR in the absence or presence of Torin2 in a kinase assay system, followed by western blot analysis. (C) Immunoprecipitated raptor, rictor and mTOR complexes were incubated with recombinant InsR in the absence or presence of Torin2 in a kinase assay system, followed by western blot analysis. (D) Rictor, mTOR and ATM were immunoprecipitated in a high salt buffer. The immunoprecipitates were incubated with kinase-dead IGF-IR (KD-IGF-IR, K1003R) in a kinase assay system, followed by western blot analysis. (E) Rictor, mTOR and ATM were immunoprecipitated in high salt buffer. The immunoprecipitates were incubated with KD-InsR (K1018A) in a kinase assay system, followed by western blot analysis. (F) Flag-tagged wild-type mTOR (wt-mTOR) or KD-mTOR (S2035T/D2357E) were overexpressed in MDA-MB-453 cells, followed by immunoprecipitation with anti-FLAG antibody. The immunoprecipitates were incubated with Akt2 or KD-IGF-IR (K1003R) in a kinase assay system, followed by western blot analysis. (G) Flag-tagged wt-mTOR or KD-mTOR were overexpressed in MDA-MB-453 cells, followed by immunoprecipitation with anti-FLAG antibody. The immunoprecipitates were incubated with Akt2 or KD-InsR (K1018A) in a kinase

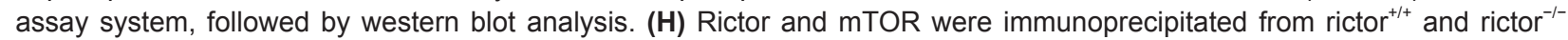
MCF-10A cells. The immunoprecipitates were incubated with kD-IGF-IR in a kinase assay system, followed by western blot analysis. (I) Rictor and mTOR were immunoprecipitated from rictor $^{+/+}$and rictor $^{-/-} \mathrm{MCF}-10 \mathrm{~A}$ cells. The immunoprecipitates were incubated with KD-InsR in a kinase assay system, followed by western blot analysis.

4E). The S2035T/D2357E mutant of mTOR is kinase-inactive [27]. While the wild-type mTOR immunoprecipitates phosphorylated the triple tyrosine cluster in KDIGF-IR/InsR and S474 in Akt2, neither KD-IGF-IR/InsR nor Akt2 was phosphorylated by immunoprecipitates of the S2035T/D2357E mutant (Figure 4F, 4G). Moreover, the immunoprecipitates of rictor and mTOR from MCF10A cells phosphorylated KD-IGF-IR/InsR, whereas the counterparts from rictor-null MCF-10A cells did not (Figure 4H, 4I). Collectively, these data demonstrate that immunoprecipitates of rictor and mTOR can phosphorylate IGF-IR/InsR independent of the intrinsic kinase 
activity of IGF-IR/InsR.

Moreover, we used recombinant mTOR and IGF-IR/ InsR to detect the kinase activity of mTOR towards IGFIR/InsR in cell-free system. While IGF-IR/InsR exhibited autophosphorylation, the full-length mTOR further enhanced IGF-IR/InsR phosphorylation (Figure 5A, 5B). Also, IGF-IR phosphorylation was enhanced by another recombinant mTOR (1362aa-end) prepared from sf21 insect cells (Supplementary information, Figure S8). Moreover, we used recombinant IRS1 as a substrate of IGF-IR/InsR to detect the effect of mTOR on IGF-IR/ InsR activity. Whereas mTOR did not induce IRS1 phosphorylation, it further stimulated IGF-IR/InsR-induced IRS1 phosphorylation (Figure 5C, 5D). While Torin2 did not affect IGF-IR/InsR activity (Supplementary information, Figure S9A, S9B), it inhibited the induction of IGF-IR/InsR phosphorylation and activation by mTOR (Supplementary information, Figure S9C, S9D). Recombinant mTOR also stimulated IGF-IR/InsR kinase activity towards their peptide substrate (Figure 5E, 5F and Supplementary information, Figure S10). These data indicate that mTOR can directly phosphorylate and activate IGF-IR/InsR in cell-free system. Since mTORC2 physically associates with IGF-IR/InsR in cells, it would be mTORC2 but not mTORC1 that promotes IGF-IR/ InsR phosphorylation and activation in vivo. Previous study has demonstrated that PI3K indirectly activates mTORC2 through phosphatidylinositol 3,4,5-trisphosphate [28]. We found that the PI3K inhibitor LY294002 repressed the induction of IGF-IR/InsR phosphorylation by rapamycin and IGF (Supplementary information, Figure S11), suggesting that PI3K-mTORC2 axis positively regulates IGF-IR/InsR activation.

\section{mTOR has tyrosine kinase activity}

mTOR is usually known as a Ser/Thr kinase. One possibility is that mTOR may phosphorylate IGF-IR/InsR on Ser/Thr residues and then indirectly promote tyrosine phosphorylation. However, treatment of HepG2 cells with okadaic acid, a Ser/Thr phosphatase inhibitor, had no effect on tyrosine phosphorylation of IGF-IR/InsR, while it led to elevated Ser/Thr phsphorylation (Supplementary information, Figure S12A). Thus, it is less likely that Ser/Thr phosphorylation of IGF-IR/InsR may lead to the triple tyrosine cluster phosphorylation in IGF-IR/ InsR. Treatment of HepG2 cells with pervanadate, a tyrosine phosphatase inhibitor, led to an increase in general tyrosine phosphorylation (Supplementary information, Figure S12B). Pervanadate treatment, however, failed to rescue the defect in tyrosine phosphorylation in IGF-IR/ InsR resulting from mTOR knockdown (Supplementary information, Figure S12B), suggesting that the promo- tion of IGF-IR/InsR phosphorylation by mTOR may be not due to negative regulation of a tyrosine phosphatase.

Notably, there is a small group of dual-specificity kinases that may phosphorylate not only serine/threonine but also tyrosine [29]. To determine whether mTOR might act as a tyrosine kinase, we examined the effect of recombinant active $\mathrm{mTOR}$ protein on tyrosine phosphorylation in vitro. IGF-IR/InsR peptide, recombinant InsR protein, and a general tyrosine kinase substrate poly(Glu-Tyr) were used as substrates. First, we detected the tyrosine phosphorylation by ELISA using a $4 \mathrm{G} 10$ phospho-tyrosine antibody. Like InsR, mTOR induced tyrosine phosphorylation in the general tyrosine kinase substrate, which was significantly inhibited by Torin2 (Figure 6A). Pre-incubation of recombinant mTOR with a monoclonal anti-mTOR antibody abrogated the phosphorylation of poly(Glu-Tyr) by mTOR (Supplementary information, Figure S13A). Also, mTOR induced tyrosine phosphorylation in IGF-IR/InsR polypeptide substrate; and this induction was inhibited by Torin 2 and blocked by the monoclonal anti-mTOR antibody (Figure 6B, 6C). Moreover, mTOR induced tyrosine phosphorylation in recombinant InsR (Figure 6D). In contrast, ATM did not induce tyrosine phosphorylation in these substrates (Figure 6A, 6B, 6D). We confirmed that mTOR kinase activity was measured in the linear range of the product-time curve (Supplementary information, Figure S13B). Moreover, dot blot analysis with 4G10 showed that mTOR could induce tyrosine phosphorylation in the general tyrosine kinase substrate poly(Glu-Tyr) and IGFIR/InsR polypeptide (Figure 6E, 6F). Collectively, these data demonstrate that $\mathrm{mTOR}$ has tyrosine kinase activity. Finally, steady-state kinetic parameters of the serine/ tyrosine kinase activity of mTOR towards different substrates were determined by ELISA (Figure 6G).

In addition, we examined the tyrosine kinase activity of rictor-mTOR complex towards the general tyrosine kinase substrate poly(Glu-Tyr) and IGF-IR/InsR polypeptide. mTOR and rictor immunoprecipitates significantly induced tyrosine phosphorylation in both poly(Glu-Tyr) and IGF-IR/InsR polypeptide, which was inhibited by Torin2 (Figure 6H, 6I). In contrast, raptor immunoprecipitates failed to induce tyrosine phosphorylation in these substrates (Figure 6H, 6I). These data demonstrate that the rictor-mTOR complex has tyrosine kinase activity towards poly(Glu-Tyr) and IGF-IR/InsR.

\section{mTOR phosphorylates IGF-IR/InsR on tyrosine residues}

We then studied the effects of recombinant mTOR on the triple tyrosine cluster of IGF-IR/InsR. First, we examined the kinase activity by measuring ADP generation upon the addition of polypeptide substrate. Whereas 
A

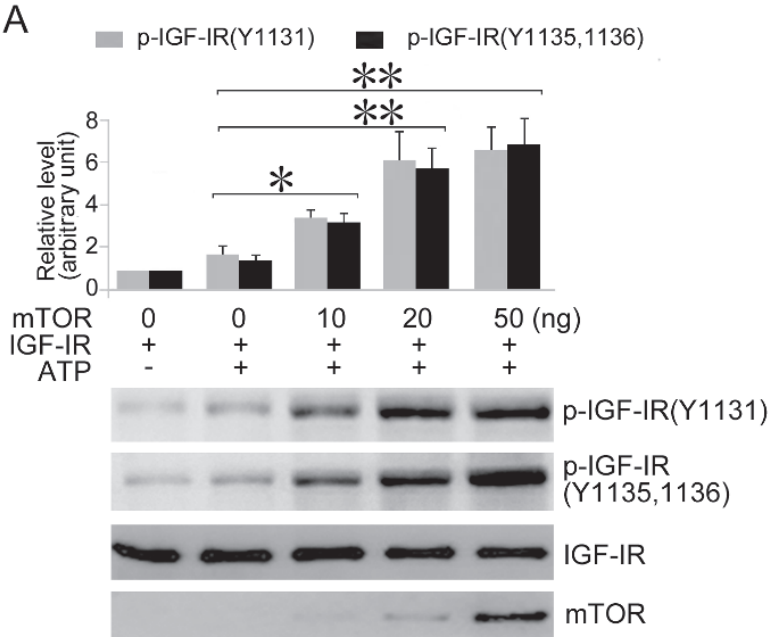

C

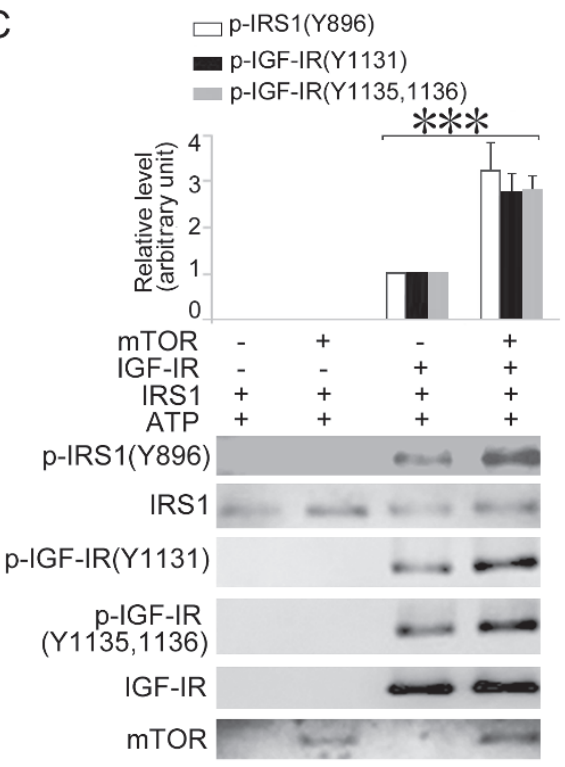

B
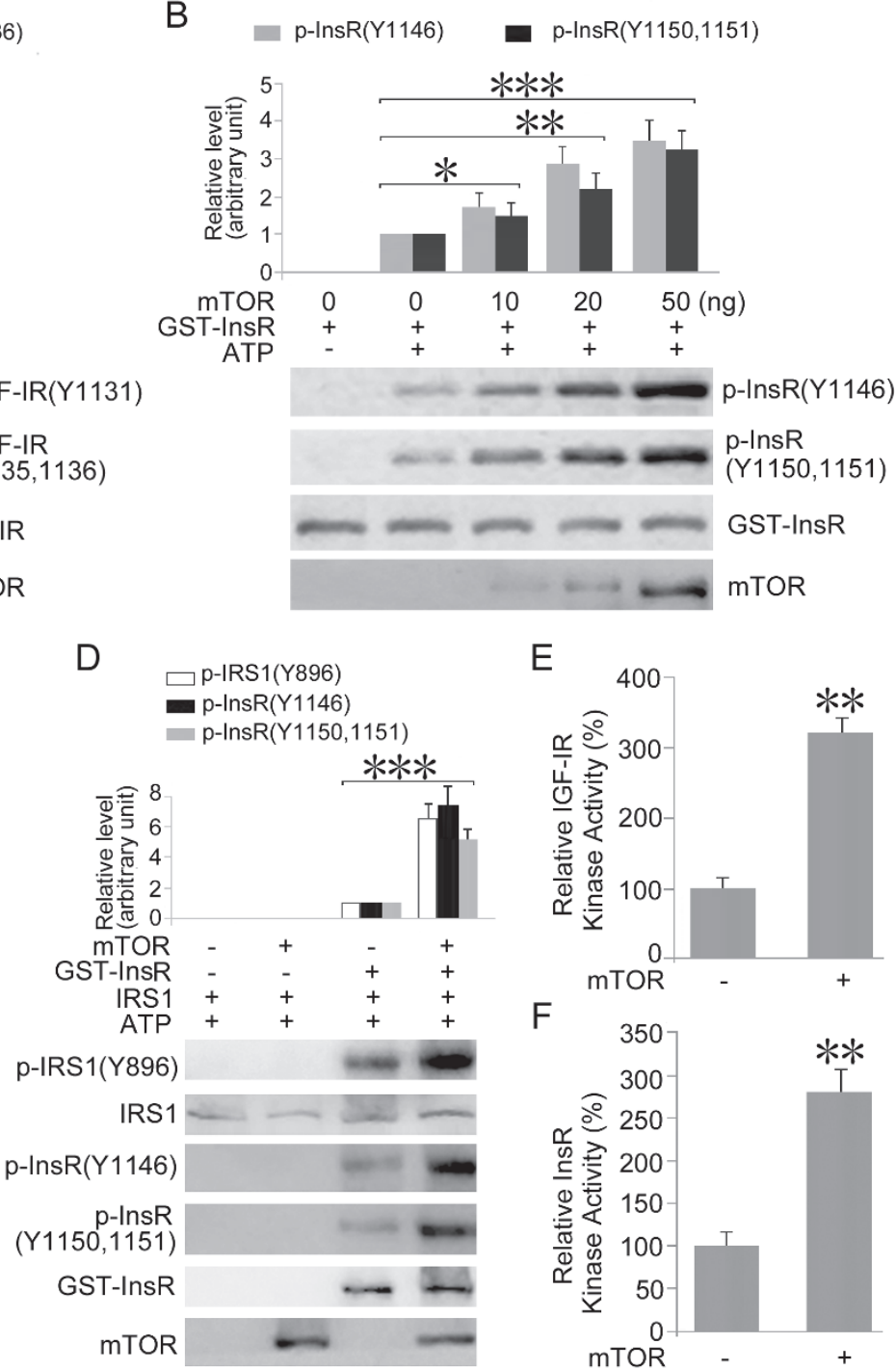

$E$

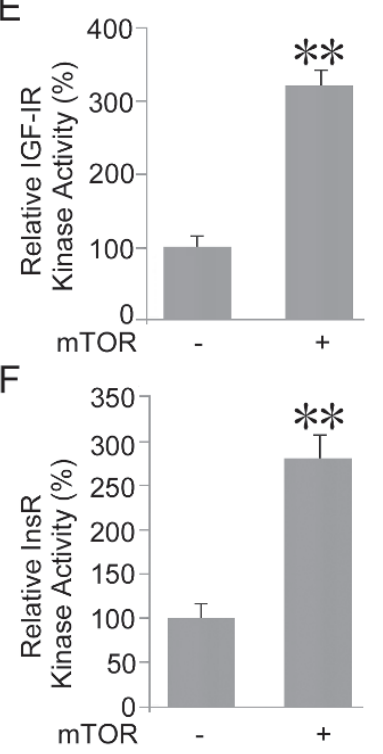

Figure 5 Recombinant mTOR promotes IGF-IR/InsR phosphorylation and activation. (A) $50 \mathrm{ng}$ of recombinant IGF-IR was incubated with or without mTOR at the indicated amount in a kinase assay system, followed by western blot analysis and quantification. Values represent mean $\pm \mathrm{SD}$. ${ }^{*} P<0.05$; ${ }^{* *} P<0.01$. (B) $50 \mathrm{ng}$ of recombinant InsR was incubated with or without $\mathrm{mTOR}$ at the indicated amount in a kinase assay system, followed by western blot analysis. Values represent mean \pm SD. $* P<0.05 ; * * P<0.01$; $* * P<0.001$. (C) $50 \mathrm{ng}$ of recombinant IRS1 was incubated with or without $50 \mathrm{ng}$ of mTOR and IGF-IR in a kinase assay system, followed by western blot analysis. Values represent mean \pm SD. ${ }^{* * *} P<0.001$. (D) $50 \mathrm{ng}$ of recombinant IRS1 was incubated with or without $50 \mathrm{ng}$ of mTOR and InsR in a kinase assay system, followed by western blot analysis. Values represent mean \pm SD. ${ }^{* * *} P<0.001$. (E) $15 \mathrm{ng}$ of recombinant IGF-IR was incubated with or without $15 \mathrm{ng}$ of mTOR protein, and $300 \mathrm{ng}$ peptide substrate of IGF-IR in a kinase assay system, followed by detection of IGF-IR kinase activity. The relative kinase activity is plotted. Values represent mean $\pm \mathrm{SD}(n=4) . * * P<0.01$. (F) $15 \mathrm{ng}$ of recombinant InsR was incubated with or without $15 \mathrm{ng}$ of mTOR protein, and $300 \mathrm{ng}$ peptide substrate of InsR in a kinase assay system, followed by detection of InsR kinase activity. The relative kinase activity is plotted. Values represent mean $\pm \operatorname{SD}(n=4)$. ${ }^{* *} P<0.01$. A representative of two or more experiments is shown.

mTOR exhibited significant kinase activity towards the IGF-IR/InsR polypeptide containing the triple tyrosine cluster, the polypeptide with phosphorylation-deficient mutation of all three tyrosine residues failed to be phos- phorylated by mTOR, indicating that mTOR targets tyrosine residues within the triple tyrosine cluster (Figure 7A and Supplementary information, Figure S14A, S14B).

To determine which tyrosine residue is directly phos- 
A

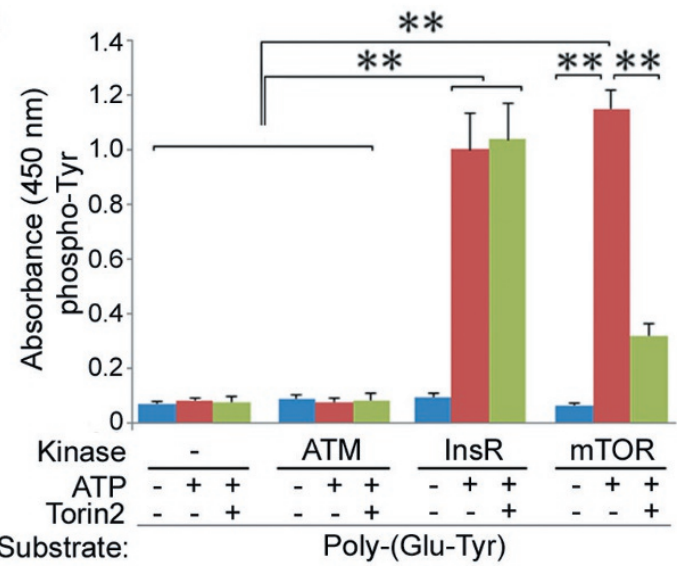

B

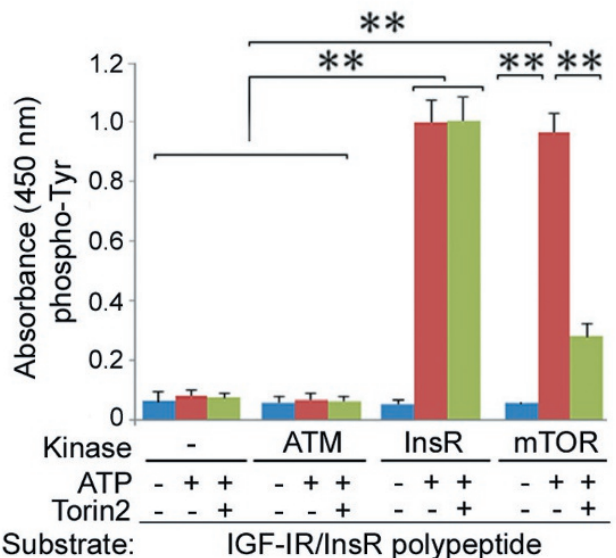

C

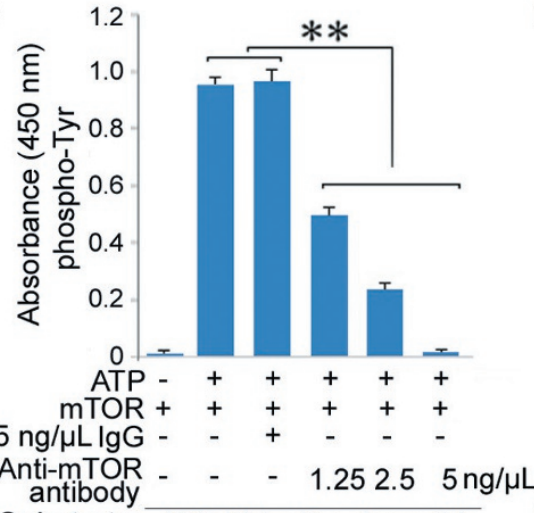

D 25

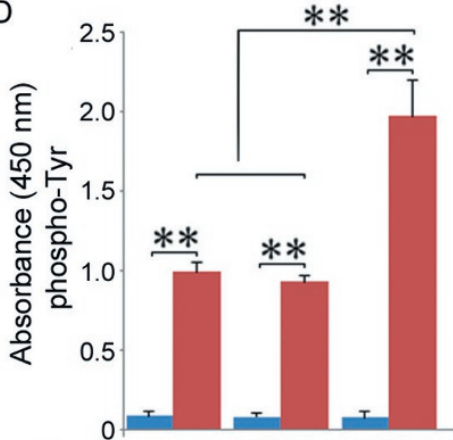

Kinase

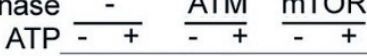

$E$

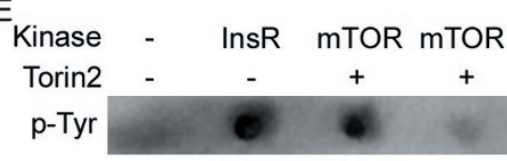

Poly-(Glu-Tyr)

$\mathrm{F}$

Kinase - InsR mTOR mTOR Torin2 $\mathrm{p}$-Tyr

Substrate: IGF-IR/InsR polypeptide Substrate:

G

\begin{tabular}{ccccc}
\hline Kinase & Peptide substrate & $\mathrm{Km}(\mu \mathrm{M})$ & $\mathrm{Kcat}\left(\mathrm{S}^{-1}\right)$ & $\mathrm{Kcat} / \mathrm{Km}\left(\mu \mathrm{M}^{-1} \mathrm{~S}^{-1}\right)$ \\
\hline \multirow{3}{*}{ mTOR } & poly-(Glu-Tyr) & $39.86 \pm 2.08$ & $2.28 \pm 0.10$ & $0.057 \pm 0.001$ \\
& IGF-IR/InsR & $27.64 \pm 2.73$ & $1.24 \pm 0.10$ & $0.045 \pm 0.001$ \\
& Akt(S473) & $65.22 \pm 11.89$ & $4.71 \pm 1.13$ & $0.072 \pm 0.005$ \\
\multirow{2}{*}{ InsR } & poly-(Glu-Tyr) & $45.36 \pm 2.13$ & $2.42 \pm 0.19$ & $0.053 \pm 0.001$ \\
& IGF-IR/InsR & $16.03 \pm 1.21$ & $2.49 \pm 0.32$ & $0.155 \pm 0.003$ \\
\hline
\end{tabular}

$\mathrm{H}$

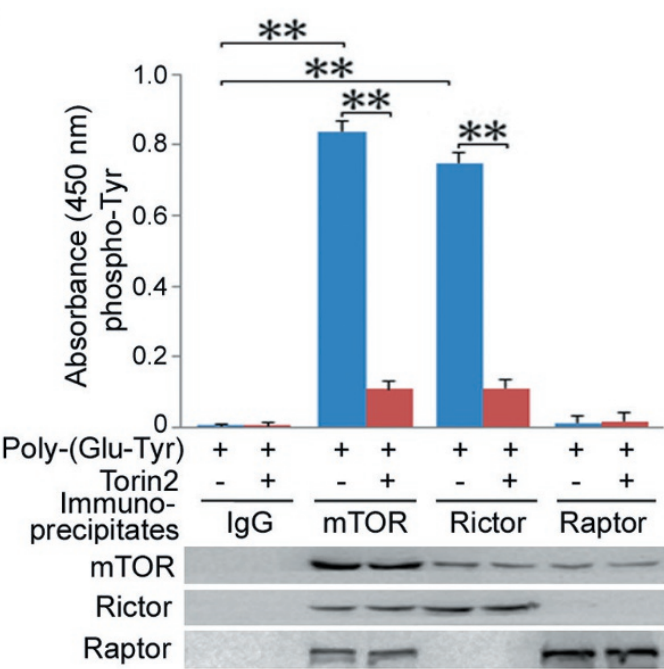

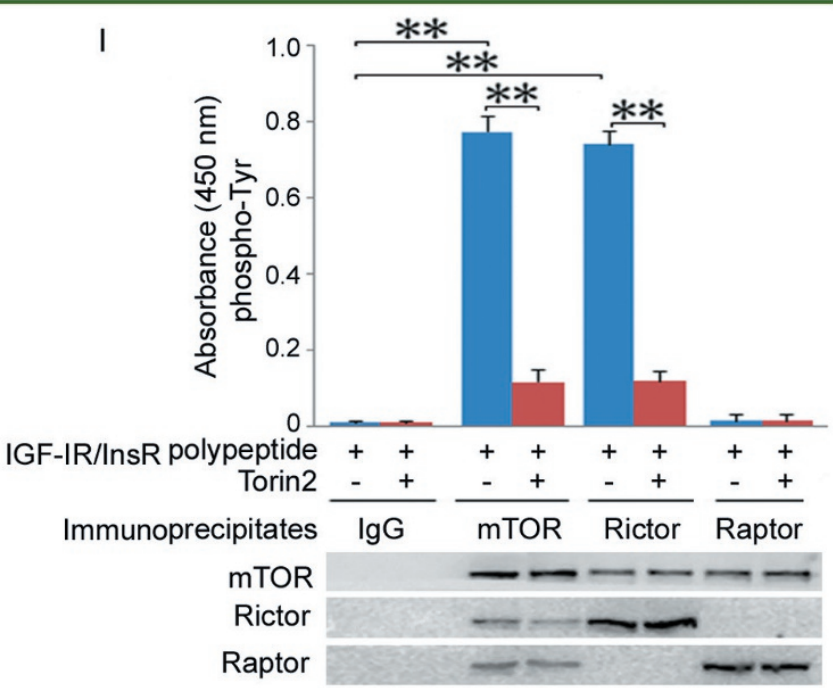


Figure 6 mTOR exhibits tyrosine kinase activity and phosphorylates tyrosine residues in IGF-IR/InsR. (A-D) In vitro kinase assays. Tyrosine phosphorylation was detected by ELISA with $4 \mathrm{G} 10$ antibody. The plates in A were coated with poly(Glu-Tyr). The plates in B were coated with peptide fragment shared by IGF-IR and InsR (TRDIYETDYYRKG). The plates in C were coated with peptide fragment shared by IGF-IR and InsR (TRDIYETDYYRKG), followed by kinase assay in the presence of normal lgG or anti-mTOR antibody. The plates in $\mathbf{D}$ were coated with recombinant InsR protein. Values represent mean $\pm \mathrm{SD}(n=3)$. $* * P<0.001$. (E) Dot blot analysis of tyrosine phosphorylation in poly(Glu-Tyr) peptide reacted with or without InsR, mTOR and Torin2. (F) Dot blot analysis of tyrosine phosphorylation in IGF-IR/InsR peptide reacted with or without InsR, mTOR and Torin2. (G) Steady-state kinetic parameters of mTOR and InsR towards individual peptide substrates determined by ELISA with 4 G10 or p-Akt (S473) antibody. (H) Immunoprecipitated raptor, rictor and mTOR complexes were incubated with poly(Glu-Tyr) in the absence or presence of Torin2 in a kinase assay system, followed by ELISA detection of tyrosine phosphorylation with $4 \mathrm{G} 10$. $* * P<0.001$. (I) Immunoprecipitated raptor, rictor and mTOR complexes were incubated with IGF-IR/InsR peptide in the absence or presence of Torin2 in a kinase assay system, followed by ELISA detection of tyrosine phosphorylation with $4 \mathrm{G} 10$. $* * P<0.001$. A representative of two or more experiments is shown.

phorylated by mTOR, we synthesized three peptides with phosphorylation-deficient mutation of each tyrosine residue in the triple tyrosine cluster. mTOR had significantly less kinase activity towards the substrates with phosphorylation-deficient mutation of Y1131 and/or Y1136 for IGF-IR, Y1146 and/or Y1151 for InsR, while the kinase activity was intact towards the substrate with phosphorylation-deficient mutation of Y1135 for IGF-IR or Y1150 for InsR (Figure 7A and Supplementary information, Figure S14A, S14B). Although both Y1131 (IGF-IR)/ Y1146 (InsR) and Y1136 (IGF-IR)/Y1151 (InsR) appeared to be residues that could be phosphorylated by mTOR, mutation of Y1131 (IGF-IR)/Y1146 (InsR) alone led to drastic loss of response to mTOR (Figure 7A and Supplementary information, Figure S14A, S14B), suggesting that phosphorylation of Y1131 (IGF-IR)/Y1146 (InsR) and Y1136 (IGF-IR)/Y1151 (InsR) residues might be co-operative processes.

The phosphorylation of IGF-IR/InsR by mTOR was further determined by ELISA. Both mTOR and InsR significantly induced Y1131 (IGF-IR)/Y1146 (InsR) phosphorylation (Figure 7B). While Y1135 (IGF-IR)/Y1150 (InsR) phosphorylation was induced by InsR, it was not induced by mTOR (Figure 7C). ELISA detection of IGFIR/InsR peptide and mutated peptides demonstrated that mTOR induced Y1135/1136 (IGF-IR)/Y1150/1151 (InsR) phosphorylation (Figure 7D). Whereas mutation of residue Y1136 (IGF-IR)/Y1151 (InsR) had no effect on the induction of Y1131 (IGF-IR)/Y1146 (InsR) phosphorylation by mTOR (Supplementary information, Figure S14C), phosphorylation-deficient mutation of Y1131 (IGF-IR)/Y1146 (InsR) led to significant decrease in the phosphorylation of Y1136 (IGF-IR)/Y1151 (InsR) by mTOR (Figure 7E). These data suggest that effective phosphorylation of Y1136 (IGF-IR)/Y1151 (InsR) by mTOR is dependent on Y1131 (IGF-IR)/Y1146 (InsR). Mutation of T1133 (IGF-IR)/T1148 (InsR) or D1134 (IGF-IR)/D1149 (InsR) had no effect on phosphorylation by mTOR (Supplementary information, Figure S14D, S14E). Dot blot analyses also supported these findings (Figure 7F-7I).

Phosphorylation of Y1131 in IGF-IR and Y1146 in InsR is essential for the activation of IGF-IR/InsR by mTOR

To determine whether phosphorylation of Y1131/1136 residues in IGF-IR is essential for the activation of IGFIR by mTOR, we mutated these two residues into phenylalanie through site-directed mutagenesis of IGF-IR construct, followed by transfection of wild type IGF-IR (wtIGF-IR) or mutated IGF-IR into MDA-MB-453 cells and immunoprecipitation of IGF-IR. The immunoprecipitated IGF-IR was incubated with recombinant mTOR and IRS1 in kinase assay system. Given that Y1135 is an autophosphorylation site, the phosphorylation of Y1135 may serve as a read-out for IGF-IR activity. Indeed, the phosphorylation of Y1135 residue in wtIGF-IR was enhanced by mTOR (Figure 8A). mTOR failed to induce the phosphorylation of Y1135 in IGF-IR with mutated Y1131/1136 (Figure 8A). Thus, mTOR may directly phosphorylate Y1131/1136, which in turn activates IGFIR and stimulates autophosphorylation of the triple tyrosine cluster leading to full activation of IGF-IR. Whereas mTOR itself did not induce IRS1 phosphorylation, it further stimulated the induction of IRS1 phosphorylation by wtIGF-IR (Figure 8A). However, mTOR did not induce IRS1 phosphorylation in the presence of mutated IGF-IR (Figure 8A). Notably, phosphorylation-deficient mutation of Y1131 alone abrogated the induction of Y1135/1136 phosphorylation and IGF-IR activation by mTOR, which was consistent with the requirement of Y1131 phosphorylation for efficient induction of Y1136 phosphorylation by mTOR.

To confirm that mTOR could phosphorylate IGF-IR on tyrosine residues as opposed to stimulating IGF-IR's autophosphorylating activity, we took advantage of the KD-IGF-IR (K1003R) to detect the effects of mTOR 
protein on IGF-IR phosphorylation and activity. KDIGF-IR failed to undergo autophosphorylation, nor did it phosphorylate IRS1 (Figure 8A). mTOR could phosphorylate Y1131, but not Y1135 in KD-IGF-IR (Figure 8A). However, a positive signal was detected by the antibody that recognized either Y1135 or Y1136 phosphorylation, indicating that mTOR could phosphorylate Y1136 in KD-IGF-IR (Figure 8A). Moreover, mass spectrometric analyses of KD-IGF-IR demonstrated that mTOR induced Y1131, Y1136, Y984 and Y1095 phosphorylation
(Supplementary information, Figure S15). However, phosphorylation-deficient mutation of both Y984 and Y1095 had no effect on mTOR-induced phosphorylation of Y1131/1136 and activation of IGF-IR (data not shown). Taken together, these data indicate that phosphorylation of Y1131/1136, especially Y1131, in IGF-IR is essential for the activation of IGF-IR by mTOR.

Similar to IGF-IR, mTOR enhanced phosphorylation of the triple tyrosine cluster in InsR. However, mTOR failed to induce the phosphorylation of Y1150 in InsR

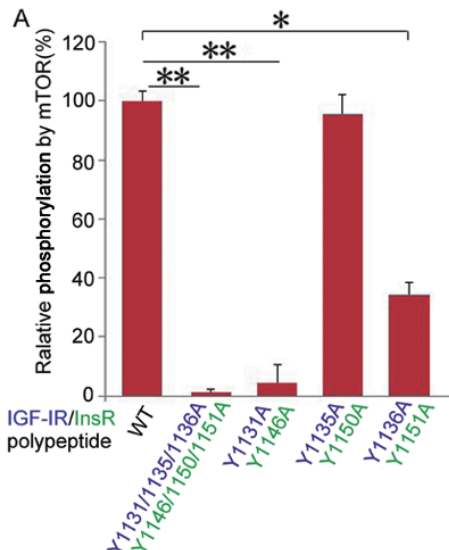

E

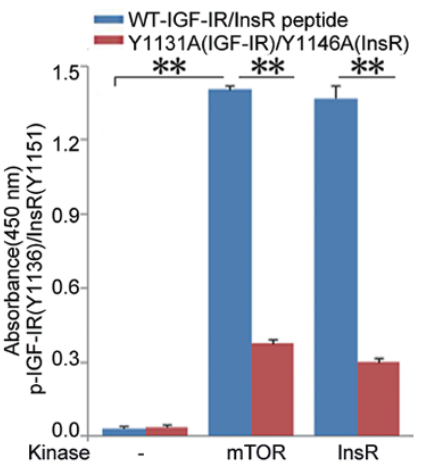

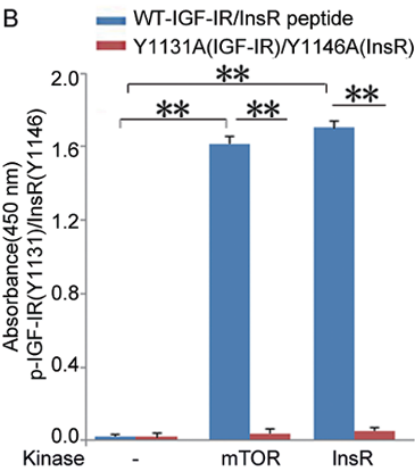
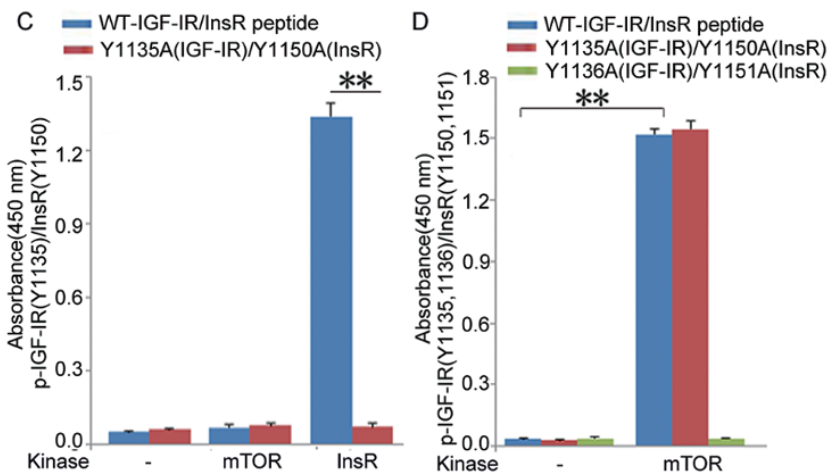

F
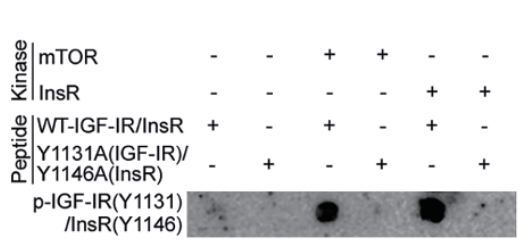
G ఖ্ّ 空| InsR
ฆ|WT-IGF-IR/InsR Y1135A(IGF-IR) ه / Y1150A(InsR) p-IGF-IR(Y1135) Y1150(InsR)

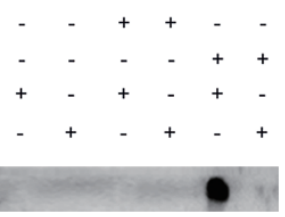

$\mathrm{H}$

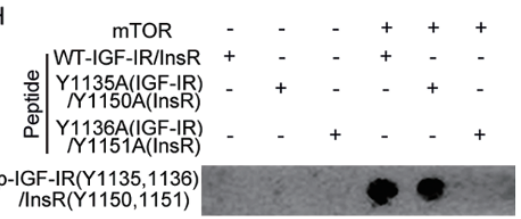

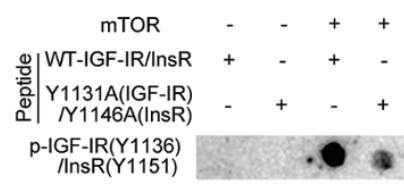

Figure 7 mTOR phosphorylates tyrosine residues in IGF-IR/InsR. (A) In an in vitro kinase assay system, $15 \mathrm{ng}$ of recombinant mTOR was incubated with $300 \mathrm{ng}$ of peptide shared by IGF-IR and InsR (TRDIYETDYYRKG) with or without mutations within the triple tyrosine cluster. WT, wild-type. The mutated residues in IGF-IR and InsR are indicated by blue labels and green labels, respectively. The relative phosphorylation by mTOR kinase was plotted. The responsiveness to $\mathrm{mTOR}$ kinase in WT group is set as $100 \%$. Values represent mean \pm SD $(n=3) . * P<0.01$; ${ }^{* *} P<0.001$. (B) ELISA detection of Y1131 (IGFIR)/Y1146 (InsR) phosphorylation. Y1131A (IGF-IR)/Y1146A(InsR) mutant peptide served as a negative control. Values represent mean $\pm \mathrm{SD}(n=3) .{ }^{*} P<0.001$. (C) ELISA detection of Y1135 (IGF-IR)/Y1150 (InsR) phosphorylation. Y1135A (IGF-IR)/ $\mathrm{Y} 1150 \mathrm{~A}$ (InsR) peptide served as a negative control. Values represent mean $\pm \mathrm{SD}(n=3)$. ** $P<0.001$. (D) ELISA detection of Y1136 (IGF-IR)/Y1151 (InsR) phosphorylation. Y1136A (IGF-IR)/Y1151A (InsR) peptide served as a negative control. Values represent mean $\pm \operatorname{SD}(n=3)$. $* * P<0.001$. (E) ELISA detection of the effect of $Y 1131$ (IGF-IR)/Y1146 (InsR) on Y1136 $($ IGF-IR)/Y1151 (InsR) phosphorylation. Values represent mean $\pm \mathrm{SD}(n=3)$. ** $P<0.001$. (F) Dot blot analysis of $\mathrm{mTOR}-$ or InsR-induced Y1131 (IGF-IR)/Y1146 (InsR) phosphorylation in peptide substrates. Y1131A (IGF-IR)/Y1146A (InsR) peptide served as a negative control. (G) Dot blot analysis of mTOR- or InsR-induced Y1135 (IGF-IR)/Y1150 (InsR) phosphorylation in IGF-IR/InsR peptide. Y1135A (IGF-IR)/Y1150A (InsR) peptide served as a negative control. (H) Dot blot analysis of mTOR-induced Y1136 (IGF-IR)/Y1151 (InsR) phosphorylation. Y1136A (IGF-IR)/Y1151A (InsR) peptide served as a negative control. (I) Dot blot analysis of the effect of Y1131 (IGF-IR)/Y1146 (InsR) mutation on mTOR-induced Y1136 (IGF-IR)/Y1151 (InsR) phosphorylation. A representative of three experiments is shown. 

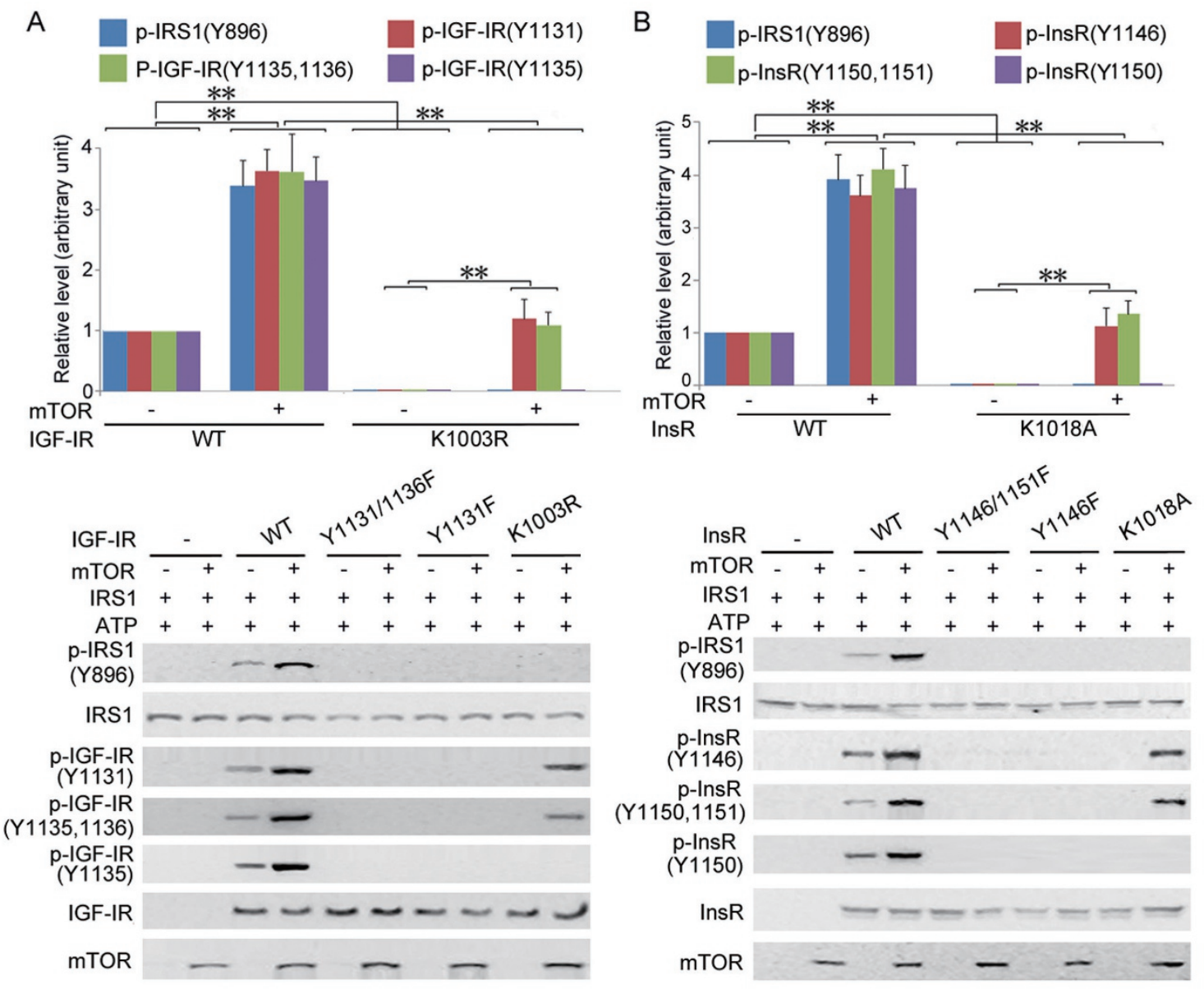

Figure 8 mTOR differentially affects the kinase activity of wild-type (WT) IGF-IR/InsR and their mutants. (A) Serum-starved MDA-MB-453 cells were transfected with WT or mutated IGF-IR (Y1131F, Y1131/1136F, kinase-dead K1003R), followed by immunoprecipitation with IGF-IR antibody. The immunoprecipitated proteins were incubated with or without recombinant mTOR and IRS1 in kinase assay system, followed by western blot analysis and quantification. Values represent mean \pm SD. $* * P<0.001$. (B) Serum-starved MDA-MB-453 cells were transfected with vectors expressing GFP-tagged WT or mutated InsR (Y1146F, Y1146/1151F, kinase-dead K1018A), followed by immunoprecipitation with GFP antibody. The immunoprecipitated proteins were incubated with recombinant mTOR and/or IRS1 in kinase assay system, followed by western blot analysis. Values represent mean $\pm \mathrm{SD}$. ${ }^{* *} P<0.001$. A representative of three experiments is shown.

with mutated Y1146/1151 (Figure 8B). Whereas mTOR itself did not induce IRS1 phosphorylation, it further stimulated the induction of IRS1 phosphorylation by wtInsR (Figure 8B). In contrast, mTOR did not stimulate IRS 1 phosphorylation in the presence of InsR (Y1146/1151F) mutant (Figure 8B). Consistent with the requirement of Y1146 phosphorylation for efficient induction of Y1151 phosphorylation by mTOR, phosphorylation-deficient mutation of Y1146 alone blunted the induction of Y1150/1151 phosphorylation and InsR activation by mTOR. Moreover, KD-InsR (K1018A) failed to undergo autophosphorylation, nor did it phosphorylate IRS1 (Figure 8B). mTOR could phosphorylate Y1146, but not Y1150 in KD-InsR (Figure 8B). However, a pos- itive signal was detected by the antibody that recognized either Y1150 or Y1151 phosphorylation, indicating that mTOR could phosphorylate Y1151 in KD-InsR (Figure 8B). Taken together, these data indicate that phosphorylation of Y1146/1151, especially Y1146, in InsR is essential for the activation of InsR by mTOR.

\section{Rictor promotes IGF-induced cellular proliferation}

The above-mentioned data demonstrate that rictor is a positive regulator of IGF and insulin signaling. Whereas rictor knockdown had little effect on HepG2 cell proliferation under serum-free condition, it inhibited IGF-Iand insulin-induced cell proliferation (Figure 9A, 9B). Overexpression of rictor significantly enhanced cell 
60
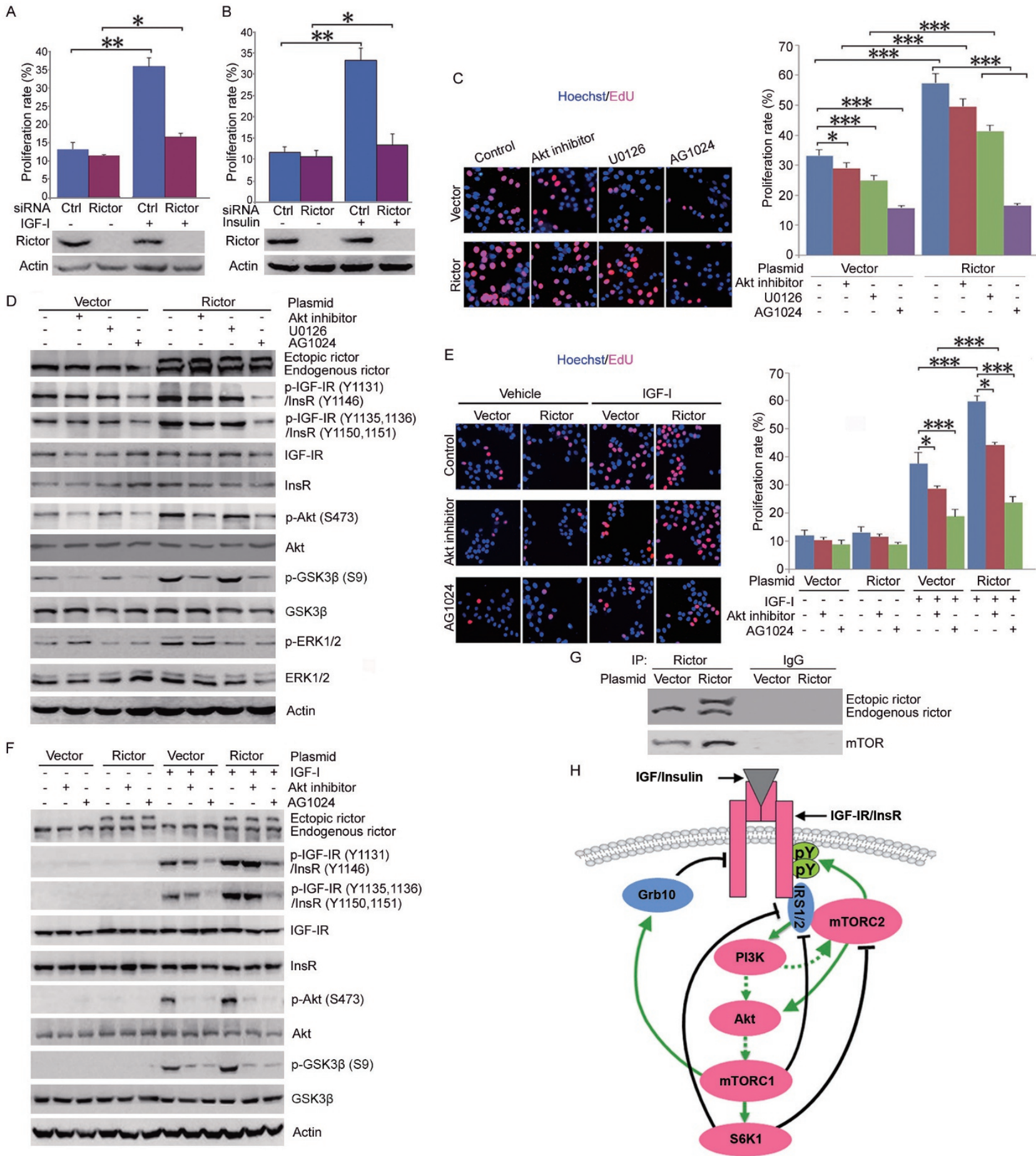

proliferation and IGF-IR/InsR signaling (Figure 9C, 9D). Treatment with the IGF-IR/InsR inhibitor AG1024 blunted the stimulation of proliferation by rictor, while MEK inhibitor moderately and Akt inhibitor slightly inhibited cell proliferation (Figure 9C, 9D). Whereas overexpression of rictor had little effect on HepG2 cells cultured in serum-free medium, it significantly stimulated IGF-I- induced cell proliferation (Figure 9E). Again, treatment with AG1024 significantly inhibited the effect of rictor on cell proliferation in the presence of IGF-I, while Akt inhibitor suppressed cell proliferation to a much lesser extent, although it significantly inhibited Akt (Figure 9E, 9F). We confirmed that overexpression of rictor did lead to increased rictor-mTOR complex (Figure 9G). 
Although rapamycin may have antiproliferative effect through mTORC1 inhibition, activation of IGF-IR/InsR may reverse the anti-proliferative effect. Indeed, combination of rapamycin and AG1024 more robustly inhibited HepG2 cell proliferation than each single agent, while rapamycin had little effect on cell proliferation (Supplementary information, Figure S16).

\section{Discussion}

In more than two decades after the discovery of mTOR and identification of it as the physical target of rapamycin $[30,31]$, there are several seminal observations that dominate thinking about mTOR biology. These observations include: (a) mTOR is an atypical serine/ threonine kinase; (b) there are two mTOR complexes, mTORC1 and mTORC2; (c) mTORC1 and mTORC2 have different targets and varied sensitivity to rapamycin; (d) mTOR functions downstream of growth factor receptors; (e) inhibition of mTORC1 may paradoxically leads to Akt and ERK phosphorylation; and (f) mTORC2 is the hydrophobic motif kinase for Akt and SGK1 [6, 32].

IGF-IR and InsR receive signals from mitogenic growth factors or hormones and stimulate cell growth, migration and metabolism. However, the molecular mechanism underlying the integration of multiple signaling elements to produce a concerted response to insulin and IGF is largely unknown. Previous studies have demonstrated that mTOR is tightly involved in IGF-IR and InsR signaling, either as an effector or regulator of several key elements in signaling pathway $[6,14,18$, 19]. mTORC1 and its substrate S6K1 promote IRS1 degradation, thereby negatively regulate IGF-IR and InsR signaling. Recently, Grb10, an adaptor protein that neg- atively regulates InsR and IGF-IR, has been identified as an mTORC1 substrate that is stabilized by mTORC $1[18$, 19]. mTORC2 positively regulates Akt and ERK, two key elements in IGF-IR/InsR signaling. In this study, we tested the hypothesis that mTOR may directly regulate InsR and IGF-IR, which input signal to Akt and ERK. Our results support this hypothesis. Our data demonstrate that rictor-mTOR promotes IGF-IR/InsR phosphorylation. In vitro kinase assays confirm that $\mathrm{mTOR}$ can directly phosphorylate and activate both IGF-IR and InsR. This finding lends support to the concept that mTOR, especially mTORC2, may act upstream of IGF-IR/InsR. In this regard, there are feedback circuits between mTORC1 and mTORC2, as shown in a working model (Figure $9 \mathrm{H})$. mTORC2 promotes ligand-induced activation of IGF-IR/InsR, leading to activation of PI3K, mTORC1 and its downstream targets. Whereas PI3K-induced PIP3 may activate mTORC2, activation of mTORC1 and its downstream targets feeds back to inhibit mTORC2 and IRS, and positively regulate Grb10. Thus, inhibition of mTORC1 may result in activation of IGF-IR/InsR signaling through repressing Grb10, the brake for IGF-IR/ InsR activation, and derepressing $\mathrm{mTORC} 2$, which in turn promotes IGF-IR/InsR phosphorylation. In contrast to the current study, a study on muscle-specific rictor knockout demonstrated that Akt T308 phosphorylation is intact during insulin stimulation [33]. However, Akt S473 phosphorylation by mTORC2 is also intact. Therefore, it appears that the phosphorylation of Akt S473 and Akt T308 is not dependent on mTORC2 in the muscle-specific rictor knockout model. The reason for this discrepancy is still unclear. It is possible there exist some compensatory mechanisms that may replace mTORC 2 in the muscle-specific rictor knockout model. Notably, there

\footnotetext{
Figure 9 Rictor stimulates IGF-I-induced cellular proliferation. (A) The effect of rictor knockdown on IGF-I-induced HepG2 cell proliferation determined by EdU labeling. The percentage of EdU-labelled cells is plotted. Values represent mean $\pm \mathrm{SD}(n=3)$. $* P<0.05 ; * *<0.01$. A representative of two independent experiments in triplicate is shown. The efficiency of rictor knockdown is determined by western blotting. (B) The effect of rictor knockdown on insulin-induced SMMC-7721 cell proliferation determined by EdU labeling. The percentage of EdU-labelled cells is plotted. Values represent mean \pm SD $(n=3)$. A representative of two independent experiments in triplicate is shown. ${ }^{*} P<0.05 ; * *<0.01$. The efficiency of rictor knockdown is determined by western blotting. (C) The effect of rictor overexpression, AG1024, Akt inhibitor IX and MEK inhibitor U0126 on HepG2 cell proliferation in serum-containing medium determined by EdU labeling. The proliferation rate was plotted. Values represent mean $\pm \mathrm{SD}(n=3)$. $* P<0.05 ; * * * P<0.001$. A representative of two independent experiments in triplicate is shown. (D) Western blot analysis of rictor expression, IGF-IR, Akt and ERK1/2 phosphorylation in $\mathbf{C}$ is shown. (E) The effect of rictor overexpression, AG1024 and Akt inhibitor IX on HepG2 cell proliferation in serum-free medium with or without IGF-I. The proliferation rate is plotted. Values represent mean $\pm \operatorname{SD}(n=3)$. $* P<0.05 ; * * * P<0.001$. A representative of two independent experiments in triplicate is shown. (F) Western blot analysis of rictor expression, IGF-IR and Akt phosphorylation in $\mathbf{E}$ is shown. (G) Immunoprecipitation of rictor from HepG2 cells transfected with or without rictor plasmid. (H) Model for mTOR in IGF-IR/InsR signaling. IRS1/2 recruits mTORC2 to IGF-IR/InsR, leading to the promotion of ligand-induced phosphorylation and activation of IGF-IR/InsR by mTORC2. As adaptor protein, IRS1/2 also connects IGF-IR/InsR to their downstream effector such as PI3K, which activates Akt/mTORC1/S6K1 axis and mTORC2. Activation of mTORC1 and S6K1 leads to feedback inhibition of IRS and rictor-mTOR complex.
} 
are reports showing that knockdown of rictor affects both Akt S473 and Akt T308 phosphorylation [6, 34], which is consistent with results showing that mTORC2 inhibitors suppress Akt T308 phosphorylation [35] and the observation in this study. These findings are in contrast to what was observed in cells from rictor knockout mice, in which the phosphorylation of Akt T308 is paradoxically elevated [9], possibly as a result of compensatory mechanisms in response to long-term loss of rictor.

Another important observation in our study is that mTOR has tyrosine kinase activity; thus, it is a novel member of dual-specificity kinases with specificity for both serine/threonine and tyrosine. IGF-IR/InsR is a substrate of mTOR tyrosine kinase. In vivo, mTOR substrate specificity is not only defined by itself, but also by the mTORC components such as raptor and rictor [36]. Although free mTOR has tyrosine kinase activity in cellfree system, only rictor-mTOR complex exhibits tyrosine kinase activity towards the general tyrosine kinase substrate and IGF-IR/InsR, whereas the raptor-mTOR complex lacks such activity. Thus, the tyrosine kinase activity of mTOR in mTORC1 is suppressed. Also, only rictor, but not raptor, interacts with IGF-IR/InsR in cells. These data together suggest that mTORC2 is responsible for activation of IGF-IR/InsR in vivo, and mTOR is a key regulator of IGF-IR and InsR signaling pathways.

IGF-IR and InsR share a triple tyrosine cluster in the kinase domain. This triple tyrosine cluster in IGF-IR and InsR undergoes autophosphorylation upon ligand binding. Phosphorylation of the triple tyrosine cluster is required for full activation of IGF-IR/InsR [26]. The current paradigm for IGF-IR/InsR activation is that ligand binding results in kinase activation and multi-site autophosphorylation, which further stimulates the kinase activity, leading to full activation of the receptor. Thus, the triple tyrosine cluster acts as an amplifier for activation of IGF-IR/InsR. Our results demonstrate that mTOR can directly phosphorylate residues in the triple tyrosine cluster. Although mTOR does not directly phosphorylate the second tyrosine residue in the triple tyrosine cluster, it can indirectly induce the phosphorylation of this tyrosine residue through IGF-IR/InsR itself. Interestingly, phosphorylation of Tyr1131 in IGF-IR or Tyr1146 in InsR appears to be essential for efficient phosphorylation of Tyr1136 in IGF-IR or Tyr1151 in InsR by mTOR and IGF-IR/InsR themselves, suggesting a cascading reaction in the triple tyrosine cluster phosphorylation. Phosphorylation of the triple tyrosine cluster by mTOR may represent a novel mechanism underlying the activation of IGF-IR/InsR. While ligand binding turns on the process of IGF-IR/InsR activation, rictor-mTOR positively regulates the extent of IGF-IR/InsR activation. We speculate that ligand binding, IGF-IR/InsR autophosphorylation and mTORC2 work in concert to fully activate IGF-IR/ InsR and produce a functional output.

IRS1 and IRS2 are known as substrates of IGF-IR/ InsR that transduce signal to downstream effectors such as PI3K and Raf. IRS1 and IRS2 also stimulate InsR activation [37]. Our current study demonstrate that IRS1/2 recruit regulators such as mTORC2 to IGFIR/InsR thereby promoting the activation of IGF-IR/ InsR. Knockdown of IRS1 or IRS2 not only abrogates ligand-induced Akt and ERK phosphorylation, but also represses ligand-induced activation of IGF-IR/InsR. These data highlight a key role of IRS1/2 in organizing signaling complexes or "signalosome" in IGF-IR/InsR pathway. mTORC1 itself is a key effector downstream of IGF-IR/InsR. However, activation of mTORC1 leads to a feedback inhibition of IRS $1 / 2$ through a mechanism involving direct phosphorylation of IRS $1 / 2$ by mTOR or indirect phosphorylation of IRS1/2 by S6K1. Phosphorylation of IRS1/2 by mTOR and S6K1 then leads to their proteasomal degradation [38-40]. Recently, it was reported that $\mathrm{mTORC} 2$ could trigger IRS1 degradation via Fbw8 stablization [24]. However, the same study also reported that depletion of SIN1 led to insufficient activation of InsR and reduced PIP3 production [24]. Our current finding highlights the first important interaction between mTORC2 and IGF-IR/InsR, and identifies IRS1/2 as important mediators of this interaction.

Rapamycin represses mTORC1 activity through negative regulation of raptor. Inhibition of mTORC1 leads to feedback derepression of rictor-mTOR thereby activating IGF-IR/InsR and Akt. Given the report that prolonged rapamycin treatment may inhibit mTORC2 assembly and Akt activation in some cell types [7], the effects of rapamycin on IGF-IR/InsR activation may also be cell type-specific. These adaptive responses may limit the efficacy of mTORC1 inhibitors in cancer therapy. Success of mTORC1 inhibition may be achieved by disrupting feedback circuits. Previous studies have demonstrated that concomitant inhibition of IGF-IR or its downstream effectors may improve the anticancer efficacy of mTORC1 inhibitors [41-43]. Our current study demonstrates a rational basis for combinating of IGF-IR/ InsR and mTORC1 inhibitors in cancer treatment. Furthermore, mTOR kinase inhibitors such as Torin1, Torin2 and PP242 may not only block mTORC1 and mTORC2 activity but also inhibit the activation of IGF-IR/InsR. This result reveals an important caveat that, in spite of the great promise ATP-competitive mTORC1/2 inhibitors hold in cancer therapy [44], insulin resistance may be a potential adverse effect of prolonged treatment with these inhibitors in cancer therapy. 


\section{Materials and Methods}

\section{Reagents}

The biochemical reagents used are as follows: rapamycin (Sigma), LY294002, U0126 and Akt inhibitor (Millipore), AICAR, IGF-I, insulin (PeproTech Inc), Torin2 (Tocris), Okadaic acid (Beyotime), recombinant human mTOR (OriGene and Millipore), recombinant human IGF-IR, InsR, Akt2 proteins (BPS Bioscience), recombinant human IRS1 protein (SignalChem), Poly(Glu-Tyr) (Cell Signaling Technology). The details of above reagents and antibodies are described in Supplementary information, Data S1.

\section{Plasmids}

The mammalian expression plasmid for human IGF-IR was a gift from Prof Wei Zhang (MD Anderson Cancer Center, USA). The plasmids for rictor, GFP-tagged human InsR, FLAG-tagged wt-mTOR and KD mTOR (S2035T/ D2357E) were purchased from Addgene.

\section{Cell culture}

Hepatoma cell lines HepG2, SMMC-7721, and the immortalized human liver cell line Chang liver, breast cancer cell line MDA-MB-453, cervical cancer cell line Hela and Human Embryonic Kidney 293 (HEK293) were all obtained from Cell Lines Bank, Chinese Academy of Science (Shanghai, China). These cells were maintained in Dulbecco Minimal Essential Medium (DMEM) containing $10 \%$ fetal bovine serum and 50 units $/ \mathrm{ml}$ of penicillin and $50 \mu \mathrm{g} / \mathrm{ml}$ streptomycin sulfate, and incubated at $37{ }^{\circ} \mathrm{C}$ in a humidified atmosphere of $5 \% \mathrm{CO}_{2}$. MCF-10A and rictor-null MCF-10A cells were purchased from Sigma, and maintained in DMEM-F12 medium containg 5\% horse serum, $2.5 \mathrm{mM}$ L-glutamine, $10 \mathrm{mg} / \mathrm{ml}$ human insulin, $0.5 \mathrm{mg} / \mathrm{ml}$ hydrocortisone, $10 \mathrm{ng} /$ $\mathrm{ml} \mathrm{EGF}$, and $100 \mathrm{ng} / \mathrm{ml}$ cholera toxin.

\section{Gene silencing and transfection}

All siRNAs were custom-synthesized products of Ribobio Co., Ltd. The siR-Ribo negative control was used as control for all siRNA experiments. The double-stranded siRNA or plasmid was dissolved in DEPC-treated or deionized water, respectively. Subconfluent proliferating cells were incubated with $50 \mathrm{nM}$ siRNA or $4 \mu \mathrm{g}$ plasmid in $2 \mathrm{~mL}$ of OPTI-MEMI Reduced Serum Medium (Life Technologies) containing Lipofectamine 2000 (Invitrogen).

\section{Western blotting}

Cells were lysed with ice-cold lysis buffer (1\% Triton X-100, $40 \mathrm{mM}$ Hepes pH 7.5, $120 \mathrm{mM} \mathrm{NaCl}, 1 \mathrm{mM}$ EDTA, $10 \mathrm{mM}$ pyrophosphate, $10 \mathrm{mM}$ glycerophosphate, $50 \mathrm{mM} \mathrm{NaF}, 0.5 \mathrm{mM}$ orthovanadate) containing protease inhibitors (PMSF, aprotinin and phosSTOP (Roche, Indianapolis, IN, USA)). Cell lysates were incubated on ice for $30 \mathrm{~min}$ and then centrifuged for $20 \mathrm{~min}$ at $12000 \times g$ to remove debris. Aliquots of proteins were boiled in $1 \times$ loading buffer for $10 \mathrm{~min}$, samples containing $30 \mu \mathrm{g}$ of total proteins were resolved by SDS-PAGE and proteins transferred to PVDF membrane (Millipore Corporation, Billerica, MA, USA). Membranes were incubated with primary antibodies overnight at $4{ }^{\circ} \mathrm{C}$ and appropriate HRP-secondary antibodies for $1 \mathrm{~h}$ at room temperature. Detection was performed with chemiluminescent agents. Images were gathered by Alpha Innotech's FluorChem imaging system. Densitometric analysis of blots was performed with
ImageJ.

\section{Immunoprecipitation and pull-down assays}

Protein $\mathrm{G}$ agarose loaded with the indicated antibody was incubated with total cellular proteins overnight at $4{ }^{\circ} \mathrm{C}$. After three washes, bound proteins were used for in vitro kinase assays or analyzed by western blotting. The immunoprecipitation and GST pull-down assays are described in detatil in Supplementary information, Data S1.

\section{Site-directed mutagenesis}

The QuikChange Kit (Stratagene) was used for site-directed mutagenesis. Point mutations of IGF-IR and InsR were introduced in Supplementary information, Data S1. All mutations were verified by DNA sequencing.

\section{In vitro kinase assays}

Kinases and their substrates were incubated in vitro in the absence or presense of ATP. The kinase activity was measured in western blot analysis using substrate protein phosphorylation as the readout, or by EnzyChrom Kinase Assay (BioAssay Systems, Hayward, CA, USA) using ATP hydrolysis as the readout, dot blot and ELISA analysis using peptide substrates phosphorylation as the readout. These assays were described in detail in Supplementary information, Data $\mathrm{S} 1$.

\section{Cell proliferation assay}

Cell proliferation was detected by EdU labeling. In addition, all cells were labeled by Hoechst 33342 . The cell proliferation rate was determined by the percentage of EdU-labeled cells.

\section{Statistical analysis}

One-way ANOVA with post hoc tests was used in statistical analysis. All statistical tests were two-tailed. Differences were considered statistically significant if $P<0.05$.

\section{Acknowledgments}

We thank Prof Wei Zhang (Cancer Genomics Core Laboratory, MD Anderson Cancer Center, USA) for generously providing pcDNA3.1-IGF-IR plasmid. This work was supported by the intramural research funding from West China Hospital, Sichuan University, China.

\section{References}

1 Kim J, Kundu M, Viollet B, Guan KL. AMPK and mTOR regulate autophagy through direct phosphorylation of Ulk1. Nat Cell Biol 2011; 13:132-141.

2 Laplante M, Sabatini DM. mTOR signaling in growth control and disease. Cell 2012; 149:274-293.

3 Ben-Sahra I, Howell JJ, Asara JM, Manning BD. Stimulation of de novo pyrimidine synthesis by growth signaling through mTOR and S6K1. Science 2013; 339:1323-1328.

4 Robitaille AM, Christen S, Shimobayashi M, et al. Quantitative phosphoproteomics reveal mTORC1 activates de novo pyrimidine synthesis. Science 2013; 339:1320-1323.

5 Jacinto E, Loewith R, Schmidt A, et al. Mammalian TOR 
complex 2 controls the actin cytoskeleton and is rapamycin insensitive. Nat Cell Biol 2004; 6:1122-1128.

6 Sarbassov DD, Guertin DA, Ali SM, Sabatini DM. Phosphorylation and regulation of Akt/PKB by the rictor-mTOR complex. Science 2005; 307:1098-1101.

7 Sarbassov DD, Ali SM, Sengupta S, et al. Prolonged rapamycin treatment inhibits mTORC2 assembly and Akt/PKB. Mol Cell 2006; 22:159-168.

8 Garcia-Martinez JM, Alessi DR. mTOR complex 2 (mTORC2) controls hydrophobic motif phosphorylation and activation of serum- and glucocorticoid-induced protein kinase 1 (SGK1). Biochem J 2008; 416:375-385.

9 Guertin DA, Stevens DM, Thoreen CC, et al. Ablation in mice of the mTORC components raptor, rictor, or mLST8 reveals that mTORC2 is required for signaling to Akt-FOXO and PKCalpha, but not S6K1. Dev Cell 2006; 11:859-871.

10 Dibble CC, Asara JM, Manning BD. Characterization of Rictor phosphorylation sites reveals direct regulation of mTOR complex 2 by S6K1. Mol Cell Biol 2009; 29:5657-5670.

11 Julien LA, Carriere A, Moreau J, Roux PP. mTORC1-activated S6K1 phosphorylates Rictor on threonine 1135 and regulates mTORC2 signaling. Mol Cell Biol 2010; 30:908-921.

12 Liu P, Gan W, Inuzuka H, et al. Sin1 phosphorylation impairs mTORC2 complex integrity and inhibits downstream Akt signalling to suppress tumorigenesis. Nat Cell Biol 2013; 15:1340-1350.

13 Kinkade CW, Castillo-Martin M, Puzio-Kuter A, et al. Targeting AKT/mTOR and ERK MAPK signaling inhibits hormone-refractory prostate cancer in a preclinical mouse model. $J$ Clin Invest 2008; 118:3051-3064.

14 O'Reilly KE, Rojo F, She QB, et al. mTOR inhibition induces upstream receptor tyrosine kinase signaling and activates Akt. Cancer Res 2006; 66:1500-1508.

15 Brown J, Jones EY, Forbes BE. Keeping IGF-II under control: lessons from the IGF-II-IGF2R crystal structure. Trends Biochem Sci 2009; 34:612-619.

16 Pollak M. The insulin and insulin-like growth factor receptor family in neoplasia: an update. Nat Rev Cancer 2012; 12:159169.

17 Pollak M. Insulin and insulin-like growth factor signalling in neoplasia. Nat Rev Cancer 2008; 8:915-928.

18 Hsu PP, Kang SA, Rameseder J, et al. The mTOR-regulated phosphoproteome reveals a mechanism of mTORC1-mediated inhibition of growth factor signaling. Science 2011; 332:13171322.

19 Yu Y, Yoon SO, Poulogiannis G, et al. Phosphoproteomic analysis identifies Grb10 as an mTORC1 substrate that negatively regulates insulin signaling. Science 2011; 332:13221326.

20 Dai N, Christiansen J, Nielsen FC, Avruch J. mTOR complex 2 phosphorylates IMP1 cotranslationally to promote IGF2 production and the proliferation of mouse embryonic fibroblasts. Genes Dev 2013; 27:301-312.

21 Dai N, Rapley J, Angel M, Yanik MF, Blower MD, Avruch J. mTOR phosphorylates IMP2 to promote IGF2 mRNA translation by internal ribosomal entry. Genes Dev 2011; 25:11591172.

22 Cybulski N, Polak P, Auwerx J, Ruegg MA, Hall MN. mTOR complex 2 in adipose tissue negatively controls whole-body growth. Proc Natl Acad Sci USA 2009; 106:9902-9907.

23 Kumar A, Lawrence JC, Jr., Jung DY, et al. Fat cell-specific ablation of rictor in mice impairs insulin-regulated fat cell and whole-body glucose and lipid metabolism. Diabetes 2010; 59: 1397-1406.

24 Kim SJ, DeStefano MA, Oh WJ, et al. mTOR complex 2 regulates proper turnover of insulin receptor substrate-1 via the ubiquitin ligase subunit Fbw8. Mol Cell 2012; 48:875-887.

25 Hernandez-Sanchez C, Blakesley V, Kalebic T, Helman L, LeRoith D. The role of the tyrosine kinase domain of the insulin-like growth factor-I receptor in intracellular signaling, cellular proliferation, and tumorigenesis. J Biol Chem 1995; 270:29176-29181.

26 Lopaczynski W, Terry C, Nissley P. Autophosphorylation of the insulin-like growth factor I receptor cytoplasmic domain. Biochem Bioph Res Commun 2000; 279:955-960.

27 Vilella-Bach M, Nuzzi P, Fang Y, Chen J. The FKBP12-rapamycin-binding domain is required for FKBP12-rapamycin-associated protein kinase activity and G1 progression. $J$ Biol Chem 1999; 274:4266-4272.

28 Gan X, Wang J, Su B, Wu D. Evidence for direct activation of mTORC2 kinase activity by phosphatidylinositol 3,4,5-trisphosphate. J Biol Chem 2011; 286:10998-11002.

29 Dhanasekaran N, Premkumar Reddy E. Signaling by dual specificity kinases. Oncogene 1998; 17:1447-1455.

30 Heitman J, Movva NR, Hall MN. Targets for cell cycle arrest by the immunosuppressant rapamycin in yeast. Science 1991; 253:905-909.

31 Brown EJ, Albers MW, Shin TB, et al. A mammalian protein targeted by G1-arresting rapamycin-receptor complex. Nature 1994; 369:756-758.

32 Hong F, Larrea MD, Doughty C, Kwiatkowski DJ, Squillace R, Slingerland JM. mTOR-raptor binds and activates SGK1 to regulate p27 phosphorylation. Mol Cell 2008; 30:701-711.

33 Bentzinger CF, Romanino K, Cloetta D, et al. Skeletal muscle-specific ablation of raptor, but not of rictor, causes metabolic changes and results in muscle dystrophy. Cell Metab 2008; 8:411-424.

34 Hresko RC, Mueckler M. mTOR. RICTOR is the Ser473 kinase for Akt/protein kinase B in 3T3-L1 adipocytes. $J$ Biol Chem 2005; 280:40406-40416.

35 Feldman ME, Apsel B, Uotila A, et al. Active-site inhibitors of mTOR target rapamycin-resistant outputs of mTORC1 and mTORC2. PLoS Biol 2009; 7:e38.

36 Hoeffer CA, Klann E. mTOR signaling: at the crossroads of plasticity, memory and disease. Trends Neurosci 2010; 33:6775.

37 Niessen M, Jaschinski F, Item F, McNamara MP, Spinas GA, Trub T. Insulin receptor substrates 1 and 2 but not Shc can activate the insulin receptor independent of insulin and induce proliferation in CHO-IR cells. Exp Cell Res 2007; 313:805815.

38 Harrington LS, Findlay GM, Gray A, et al. The TSC1-2 tumor suppressor controls insulin-PI3K signaling via regulation of IRS proteins. J Cell Biol 2004; 166:213-223.

39 Um SH, Frigerio F, Watanabe M, et al. Absence of S6K1 protects against age- and diet-induced obesity while enhancing insulin sensitivity. Nature 2004; 431:200-205.

40 Briaud I, Dickson LM, Lingohr MK, McCuaig JF, Lawrence 
JC, Rhodes CJ. Insulin receptor substrate-2 proteasomal degradation mediated by a mammalian target of rapamycin (mTOR)-induced negative feedback down-regulates protein kinase B-mediated signaling pathway in beta-cells. $J$ Biol Chem 2005; 280:2282-2293.

41 Wei F, Liu Y, Bellail AC, et al. K-Ras mutation-mediated IGF-1-induced feedback ERK activation contributes to the rapalog resistance in pancreatic ductal adenocarcinomas. Cancer Lett 2012; 322:58-69.

42 Naing A, LoRusso P, Fu S, et al. Insulin growth factor-receptor (IGF-1R) antibody cixutumumab combined with the
mTOR inhibitor temsirolimus in patients with refractory Ewing's sarcoma family tumors. Clin Cancer Res 2012; 18:26252631.

43 Schwartz GK, Tap WD, Qin LX, et al. Cixutumumab and temsirolimus for patients with bone and soft-tissue sarcoma: a multicentre, open-label, phase 2 trial. Lancet Oncol 2013; 14:371-382.

44 Janes MR, Limon JJ, So L, et al. Effective and selective targeting of leukemia cells using a TORC1/2 kinase inhibitor. Nat Med 2010; 16:205-213.

(Supplementary information is linked to the online version of the paper on the Cell Research website.) 